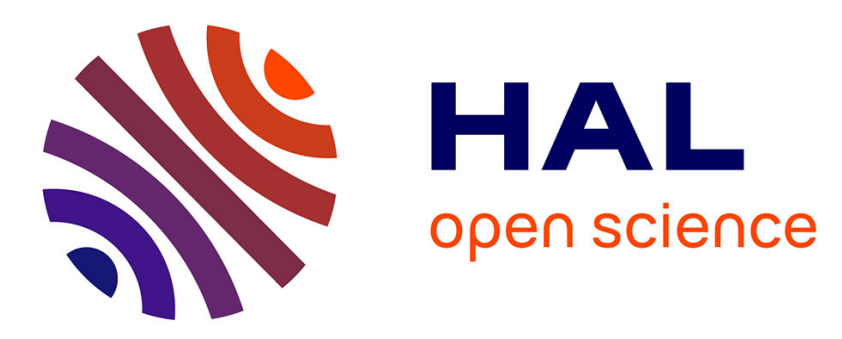

\title{
Efficient stress relaxation in molecular dynamics simulations of semiflexible $\mathbf{n}$-alkanes
}

T. Mülders, S. Toxvaerd, G. Kneller

\section{To cite this version:}

T. Mülders, S. Toxvaerd, G. Kneller. Efficient stress relaxation in molecular dynamics simulations of semiflexible n -alkanes. Physical Review E , 1998, 58 (5), pp.6766-6780. 10.1103/PhysRevE.58.6766 . hal-02155551

\section{HAL Id: hal-02155551 \\ https://hal.science/hal-02155551}

Submitted on 13 Jun 2019

HAL is a multi-disciplinary open access archive for the deposit and dissemination of scientific research documents, whether they are published or not. The documents may come from teaching and research institutions in France or abroad, or from public or private research centers.
L'archive ouverte pluridisciplinaire HAL, est destinée au dépôt et à la diffusion de documents scientifiques de niveau recherche, publiés ou non, émanant des établissements d'enseignement et de recherche français ou étrangers, des laboratoires publics ou privés. 


\title{
Efficient stress relaxation in molecular dynamics simulations of semiflexible $\boldsymbol{n}$-alkanes
}

\author{
T. Mülders \\ Institut für Biochemie, Rheinisch-Westfälische Technische Hochschule Aachen, Pauwelsstrasse 30, D-52057 Aachen, Germany \\ S. Toxvaerd \\ Department of Chemistry, H. C. Ørsted Institute, University of Copenhagen, Universitetsparken 5, \\ DK-2100 Copenhagen $\emptyset$, Denmark \\ G. R. Kneller \\ Centre de Biophysique Moléculaire, CNRS UPR No. 4301, Rue Charles Sadron, F-45071 Orléans, France
}

(Received 4 May 1998)

\begin{abstract}
We perform isobaric-isothermal molecular dynamics simulations of partially rigid $n$-alkanes of length 10 (10 carbon atoms) and 32, respectively. All bonds are considered as rigid. For these systems we compare molecular and atomic scaling to control the pressure in the Nosé-Andersen simulation scheme [S. Nosé, J. Chem. Phys. 81, 511 (1984); H. C. Andersen, ibid. 72, 2384 (1980)]. Atomic scaling in the presence of geometrical constraints means coupling all available degrees of freedom to the pressure bath, keeping the desired isobaricisothermal ensemble, and satisfying at the same time the geometrical constraints. The corresponding equations of motion have been derived recently [G. R. Kneller and T. Mülders, Phys. Rev. E 54, 6825 (1996)]. In contrast, no intramolecular degrees of freedom but only the center-of-mass positions are coupled to the pressure bath when the well established molecular scaling is applied. We demonstrate that coupling the intramolecular degrees of freedom to the volume dynamics (or, equivalently, to the pressure bath) strongly improves the relaxation of energy and volume for the long chains, while for the short chains atomic and molecular scalings are more or less equivalent in this respect. For the long chains we show explicitly that the barostat couples to intramolecular breathing modes when atomic scaling is used. The frequencies of these modes are found to be in excellent agreement with results from neutron scattering experiments.
\end{abstract}

[S1063-651X(98)02511-2]

PACS number(s): 02.70.Ns, 31.15.Qg, 36.20.-r

\section{INTRODUCTION}

In 1980 Andersen introduced the extended system method in order to perform molecular dynamics simulations in the isobaric-isoenthalpic ensemble [1]. Nosé showed that this concept also allows one to simulate the dynamics in the isobaric-isothermal ensemble [2], which corresponds to most experimental situations. While the original methods by Andersen and Nosé were designed for simulations of simple liquids, the generalization to the case of partially rigid molecules has been the subject of series of works in the past [3-10]. In contrast to atomic fluids, macromolecules are modeled mostly as semiflexible systems. Rigid covalent bonds are the most common examples for geometrical constraints that restrict the flexibility. The main reason to apply such constraints is that the computational efficiency is augmented by up to a factor of 4 avoiding the sampling of highfrequency motions [11]. While the concept of partial rigidity has the advantage of decreased computational costs, it also entails disadvantages such as more complicated equations of motion.

In simulations of atomic liquids, Andersen's pressure bath scales uniformly all coordinates in order to steer the pressure towards the desired value. Clearly, this procedure cannot be applied to partially rigid molecules without violating intramolecular geometrical constraints, e.g., frozen bonds. The standard approach to circumvent this difficulty is to couple only the centers of mass (c.m.) of the molecules to the barostat (molecular scaling or center-of-mass scaling) [8]. The c.m. positions can be obviously scaled without violating internal constraints. However, it can be expected that such a procedure becomes more and more inefficient with increasing size and decreasing number of simulated molecules. In the extreme case of a single very long macromolecule in a simulation box pressure control by center-of-mass scaling cannot be applied at all. In contrast to pressure control, temperature control by a Nosé thermostat can be straightforwardly combined with geometrical constraints, such that all degrees of freedom respond to the thermostat. Coupling only the c.m. momenta to the heat bath is known to be an inefficient procedure. Coupling as many degrees of freedom as possible to the thermostat improves the response of the system to temperature steering. The same is also desired for Andersen's barostat. Obviously, the partial rigidity of molecules allows them still to adapt their internal geometry to an applied pressure by changing the radius of gyration.

The basic idea in [3] was to incorporate all available degrees of freedom of geometrically constrained systems into the pressure control mechanism, while keeping the correct $N P T$ ensemble. Intramolecular stress can be expected to be more efficiently dissipated in this way. Dirac's theory of constrained Hamiltonian dynamics [12] together with projector techniques has been used to generalize Andersen's barostating mechanism in the presence of geometrical constraints. It can be expected that intramolecular relaxation mechanisms are important for pressure control in macromo- 
lecular systems with a larger proportion of internal degrees of freedom, whereas the center-of-mass scaling procedure can be applied without difficulties for systems consisting of a large number of relatively small molecules. It is the purpose of this work to demonstrate these points and to elucidate the differences between the center-of-mass scaling procedure and the atomic scaling method.

The paper is organized as follows. In Sec. II we specialize the equations of motion for partially rigid molecules given in [3] to the case of constraints that can be expressed as homogeneous functions of the Cartesian coordinates. The atomic scaling method is then compared to the equations of motion corresponding to the standard center-of-mass scaling procedure. We then apply linear response theory [14] in order to derive formulas for the energy dissipation spectra appropriate to describe the different response mechanisms to an externally applied compression or dilatation. In that way the rate of energy dissipation can be related to the power spectra of the instantaneous stress. In Sec. III we describe some technical details of the simulations of the alkane chains. In Sec. IV the simulation results are analyzed, which are obtained by the two different methods. Section $\mathrm{V}$ is devoted to a concluding discussion. In the Appendix we describe the integration scheme that has been used.

\section{THEORY}

\section{A. Equations of motion}

In the following we quote the results for NPT equations of motion in the case of geometrical constraints for atomic and molecular scaling, respectively. We introduce notation that simplifies the comparison for these different pressure control mechanisms.

\section{Atomic scaling}

The NPT equations of motion of an $N$-particle system subject to $l$ geometrical constraints presented here have been derived in [3]. Assuming the equivalence of time and ensemble averages, it has been shown that the equations of motion generate trajectories corresponding to the desired NPT ensemble. In the following we will use standard Cartesian coordinates instead of mass-weighted coordinates. The relations between various quantities expressed in these two coordinate systems have been given in Table 1 of Ref. [3].

The geometrical constraints describing idealized chemical structures as rigid bonds or planar rings can be cast in the general form

$$
\sigma^{\alpha}(\mathbf{r})=0, \quad \alpha=1, \ldots, l .
$$

If $N$ is the number of particles the constraints (1) leave $f$ $=3 N-l$ degrees of freedom for the configuration space of the system. We restrict ourselves to a system of $K$ identical molecules. The generalization to a system of different types of molecules is straightforward. The $K$ individual molecules are labeled by greek indices $\gamma=1, \ldots, K$, and the atoms in each molecule by latin indices $i=1, \ldots, n$ such that $N$ $=n K$. To facilitate a comparison with the center-of-mass scaling procedure, where different molecules are to be distinguished, it is convenient to introduce the $3 n$-dimensional subvectors $\mathbf{r}_{\gamma}$ and $\mathbf{p}_{\gamma}$, collecting the Cartesian positions and momenta of the $\gamma$ th molecule, respectively. One has $\mathbf{r}=\left(\mathbf{r}_{1}^{T}, \ldots \mathbf{r}_{K}^{T}\right)^{T}$, where $\mathbf{r}$ contains all the $3 N$ Cartesian coordinates of the $N$-particle system. From now on $\mathbf{M}_{\gamma}=\operatorname{diag}\left(m_{1}, m_{1}, m_{1}, \ldots, m_{n} m_{n}\right)$ stands for the $3 n \times 3 n$ mass matrix of each single molecule. Each molecule is subject to $n_{c}$ constraints such that $l=n_{c} K$. The $l$ constraint equations are now labeled as

$$
\sigma_{\gamma}^{\alpha}\left(\mathbf{r}_{\gamma}\right)=0, \quad \alpha=1, \ldots, n_{c}, \quad \gamma=1, \ldots, K
$$

and the equations of motion for atomic scaling read

$$
\begin{gathered}
\dot{\mathbf{r}}_{\gamma}=\mathbf{M}_{\gamma}^{-1} \mathbf{p}_{\gamma}+\frac{\dot{V}}{3 V} \mathbf{r}_{\gamma \|}, \\
\dot{\mathbf{p}}_{\gamma}=\mathbf{f}_{\gamma}+\mathbf{z}_{\gamma}-\frac{\dot{V}}{3 V} \mathbf{p}_{\gamma}-\zeta \mathbf{p}_{\gamma}-\frac{\dot{V}}{3 V} \mathbf{H}_{\gamma} \mathbf{M}_{\gamma}^{-1} \mathbf{p}_{\gamma}, \\
\dot{V}=\frac{p_{V}}{W_{V}},
\end{gathered}
$$

$$
\begin{gathered}
\dot{p}_{V}=P_{\text {inst }}^{a t m}-P_{\text {ext }}-\zeta p_{V}, \\
\dot{\zeta}=\frac{1}{W_{S}}\left(\sum_{\gamma} \mathbf{p}_{\gamma}^{T} \mathbf{M}_{\gamma}^{-1} \mathbf{p}_{\gamma}+\frac{p_{V}^{2}}{W_{V}}-(f+1) k_{B} T\right) .
\end{gathered}
$$

The $3 n$-dimensional vectors $\mathbf{f}_{\gamma}$ and $\mathbf{z}_{\gamma}$ comprise the potential forces and constraint forces acting on the atoms in molecule $\gamma$, respectively. As usual $k_{B} T$ is the Boltzmann constant times the absolute temperature and $P_{\text {ext }}$ is the external pressure imposed on the system. $W_{V}$ and $W_{S}$ are adjustable inertia parameters for the pressure and the temperature control, respectively. The volume is denoted as $V$ and $p_{V}$ is the associated momentum. A special feature of the equations of motion is the appearance of the $3 n \times 3 n$ matrices $\mathbf{H}_{\gamma}$, whose elements are given as

$$
\left(\mathbf{H}_{\gamma}\right)_{i k}=\sum_{\alpha} g_{\alpha, \gamma} \frac{\partial^{2} \sigma_{\gamma}^{\alpha}}{\partial r_{i, \gamma} \partial r_{k, \gamma}}, \quad i, k=1, \ldots, 3 n .
$$

The $g_{\alpha, \gamma}$ are related to Lagrangian multipliers associated with the momentum constraints. Introducing the matrix $\mathbf{A}_{\gamma}$ as

$$
\left(\mathbf{A}_{\gamma}\right)_{i}^{\alpha}=\frac{\partial \sigma_{\gamma}^{\alpha}}{\partial r_{\gamma}^{i}}, \quad \alpha=1, \ldots, n_{c}, \quad i=1, \ldots, 3 n
$$

which collects the constraint gradients as row vectors, the coefficients $g_{\alpha, \gamma}$ can be determined by solving the linear system of equations [3]

$$
\mathbf{A}_{\gamma} \underbrace{\mathbf{M}_{\gamma}^{-1} \mathbf{A}_{\gamma}^{T} \mathbf{g}_{\gamma}}_{-\mathbf{r}_{\gamma, \perp}}=-\mathbf{A}_{\gamma} \mathbf{r}_{\gamma}
$$


Here the $n_{c}$-dimensional vector $\mathbf{g}_{\gamma}$ contains the coefficients $g_{\alpha, \gamma}$, and $\mathbf{r}_{\gamma, \perp}$ is the projection of the position vector $\mathbf{r}_{\gamma}$ on the row space of $\mathbf{A}_{\gamma}$. Correspondingly, the vector $\mathbf{r}_{\gamma \|}$, with $\mathbf{r}_{\gamma \|} \perp \mathbf{r}_{\gamma, \perp}$, denotes the projection of $\mathbf{r}_{\gamma}$ onto the tangent space of the constraint surface, which is defined by Eq. (2). From Eq. (10) and $\mathbf{r}_{\gamma, \|}=\mathbf{r}_{\gamma}-\mathbf{r}_{\gamma, \perp}$ it follows immediately that

$$
\mathbf{A}_{\gamma} \mathbf{r}_{\gamma, \|}=\mathbf{0},
$$

i.e., $\mathbf{r}_{\gamma, \|}$ is in the null space of the matrix $\mathbf{A}_{\gamma}$. The appearance of the matrix $\mathbf{H}_{\gamma}$ given in Eq. (8) is a consequence of the momentum constraints that are to be explicitly imposed in the extended system method. The time derivation of the constraints (2) shows that the velocities $\dot{\mathbf{r}}_{\gamma}$ are in the null space of $\mathbf{A}_{\gamma}$, explicitly $\mathbf{A}_{\gamma} \dot{\mathbf{r}}_{\gamma}=\mathbf{0}$. In standard Hamiltonian (microcanonical) dynamics the Cartesian momenta divided by the corresponding masses are identical to the Cartesian velocities $\mathbf{M}_{\gamma}^{-1} \mathbf{p}_{\gamma}=\dot{\mathbf{r}}_{\gamma}$ and thus fulfill the constraints

$$
\mathbf{A}_{\gamma} \mathbf{M}_{\gamma}^{-1} \mathbf{p}_{\gamma}=\mathbf{0}
$$

It is necessary that the relations (12) remain valid also for equations of motion derived by the extended system method. Otherwise the accessible phase space of the constrained system would not be correctly assigned, which would lead to systematic deviations in ensemble averages of quantities containing the momenta, e.g., the temperature or the pressure [5]. This fact explains why only the parallel projections of the positions $\mathbf{r}_{\gamma, \|}$ can appear in Eq. (3).

Let us briefly consider the atomic pressure

$$
\begin{aligned}
P_{\text {inst }}^{a t m}= & \frac{1}{3 V}\left\{\sum_{\gamma} \mathbf{p}_{\gamma}^{T} \mathbf{M}_{\gamma}^{-1} \mathbf{p}_{\gamma}\right. \\
& \left.+\sum_{\gamma} \mathbf{r}_{\gamma}^{T}\left(\mathbf{f}_{\gamma}+\mathbf{z}_{\gamma}-\frac{\dot{V}}{3 V} \mathbf{H}_{\gamma} \mathbf{M}_{\gamma}^{-1} \mathbf{p}_{\gamma}\right)\right\} .
\end{aligned}
$$

It has been shown in [3] that the term proportional to $\mathbf{r}_{\gamma}^{T} \mathbf{H}_{\gamma} \mathbf{M}_{\gamma}^{-1} \mathbf{p}_{\gamma}$ does not contribute to the average pressure. As we will show now, this term can be omitted if the constraints can be written in the form

$$
\sigma_{\gamma}^{\alpha}\left(\mathbf{r}_{\gamma}\right)=q^{\alpha}\left(\mathbf{r}_{\gamma}\right)-q_{0}^{\alpha}=0
$$

where $q_{0}^{\alpha}$ is a fixed parameter and $q^{\alpha}\left(\mathbf{r}_{\gamma}\right)$ is a homogeneous function of degree $n_{\alpha}+1$ in the coordinates, $q^{\alpha}\left(\lambda \mathbf{r}_{\gamma}\right)$ $=\lambda^{\left(n_{\alpha}+1\right)} q^{\alpha}\left(\mathbf{r}_{\gamma}\right)$. The constraint derivatives themselves that appear in the matrix $\mathbf{A}_{\gamma}$ are homogeneous functions of degree $n_{\alpha}$. All standard constraints, such as bond constraints or bond angle constraints, are such homogeneous constraints. It is even questionable whether other constraints are physically meaningful. In the case of homogeneous constraints the term $\mathbf{r}_{\gamma}^{T} \mathbf{H}_{\gamma} \mathbf{M}_{\gamma}^{-1} \mathbf{p}_{\gamma}$ vanishes identically, as can be seen by using Euler's formula for homogeneous functions

$$
\begin{aligned}
\mathbf{r}_{\gamma}^{T} \mathbf{H}_{\gamma} \mathbf{M}_{\gamma}^{-1} \mathbf{p}_{\gamma} & =\sum_{\alpha} g_{\alpha, \gamma} \sum_{i, k}^{3 n} r_{i, \gamma} \frac{\partial^{2} \sigma_{\gamma}^{\alpha}}{\partial r_{i, \gamma} \partial r_{k, \gamma}} \frac{p_{k, \gamma}}{\left(\mathbf{M}_{\gamma}\right)_{k, k}} \\
& =\sum_{\alpha} g_{\alpha, \gamma} n_{\alpha} \sum_{k}^{3 n} \frac{\partial \sigma_{\gamma}^{\alpha}}{\partial r_{k, \gamma}} \frac{p_{k, \gamma}}{\left(\mathbf{M}_{\gamma}\right)_{k, k}}=0 .
\end{aligned}
$$

The second equality follows from the homogeneity of the constraint derivatives and the last equality is obtained from the momentum constraints (12). In the following we will always assume that we are dealing with homogeneous constraints only. In that case the atomic version of the instantaneous pressure reads

$$
P_{\text {inst }}^{a t m}=\frac{1}{3 V}\left\{\sum_{\gamma} \mathbf{p}_{\gamma}^{T} \mathbf{M}_{\gamma}^{-1} \mathbf{p}_{\gamma}+\sum_{\gamma} \mathbf{r}_{\gamma}^{T}\left(\mathbf{f}_{\gamma}+\mathbf{z}_{\gamma}\right)\right\} .
$$

This is exactly what one would get for the pressure in the case of microcanonical dynamics with constraints. Note that all forces, including the constraint forces $\mathbf{z}_{\gamma}$, contribute to the internal virial $[15,16]$.

\section{Molecular scaling}

In the case of molecular scaling only the centers of mass of the molecules are coupled to the pressure bath, yielding a different form for the equations of motion. The threedimensional center-of-mass vector of molecule $\gamma$ and its conjugate momentum vector are $\mathbf{R}_{\gamma}=\sum_{i}^{n} m_{i} \mathbf{r}_{i, \gamma} / M_{\gamma}$ and $\mathbf{P}_{\gamma}$ $=\sum_{i}^{n} \mathbf{p}_{i, \gamma}$, respectively. Here $M_{\gamma}$ denotes the total mass of each molecule, $M_{\gamma}=\sum_{i}^{n} m_{i}$, and $\mathbf{r}_{i, \gamma}$ and $\mathbf{p}_{i, \gamma}$ are the threedimensional Cartesian position and momentum vectors of atom $i$ in molecule $\gamma$. When the "piston momentum" $p_{V}$ is included in the thermostating procedure in the same way as in Eqs. (3)-(7) the equations of motion can be cast into the form (compare [7,9])

$$
\begin{gathered}
\dot{\mathbf{r}}_{\gamma}=\mathbf{M}_{\gamma}^{-1} \mathbf{p}_{\gamma}+\frac{\dot{V}}{3 V} \mathbf{I R}_{\gamma}, \\
\dot{\mathbf{p}}_{\gamma}=\mathbf{f}_{\gamma}+\mathbf{z}_{\gamma}-\frac{\dot{V}}{3 V} \frac{\mathbf{M}_{\gamma} \mathbf{I} \mathbf{P}_{\gamma}}{M_{\gamma}}-\zeta \mathbf{p}_{\gamma}, \\
\dot{V}=\frac{p_{V}}{W_{V}}, \\
\dot{p}_{V}=\frac{1}{3 V}\left\{\sum_{\gamma} \frac{\mathbf{P}_{\gamma}^{T} \mathbf{P}_{\gamma}}{M_{\gamma}}+\sum_{\gamma} \mathbf{R}_{\gamma}^{T} \mathbf{F}_{\gamma}\right\}-P_{e x t}-\zeta p_{V}, \\
\dot{\zeta}=\frac{1}{W_{s}}\left(\sum_{\gamma} \mathbf{p}_{\gamma}^{T} \mathbf{M}_{\gamma}^{-1} \mathbf{p}_{\gamma}+\frac{p_{V}^{2}}{W_{V}}-(f+1) k_{B} T\right) .
\end{gathered}
$$

The $(3 n \times 3)$-dimensional matrix $\mathbf{I}$ is introduced to take care of the correct dimensionality in the formulas relating vectors as $\mathbf{r}_{\gamma}$ and $\mathbf{R}_{\gamma}$,

$$
\mathbf{I}^{T}=\underbrace{[\mathbf{1 , \ldots , 1}]}_{n \text { times }}
$$

where 1 is the $3 \times 3$ unit matrix. The molecular version of the instantaneous pressure can be obtained from the equation of motion for $p_{V}$, writing $\dot{p}_{V}=P_{i n s t}^{m o l}-P_{e x t}-\zeta p_{V}$ : 
TABLE I. Atomic scaling.

\begin{tabular}{ccc}
\hline \hline Virtual variable & Real variable & Connection \\
\hline $\boldsymbol{\rho}_{\gamma}$ & $\mathbf{r}_{\gamma}$ & $\mathbf{r}_{\gamma}=V^{1 / 3} \boldsymbol{\rho}_{\gamma}$ \\
$\boldsymbol{\pi}_{\gamma}$ & $\mathbf{p}_{\gamma}$ & $\mathbf{p}_{\gamma}=V^{-1 / 3} s^{-1} \boldsymbol{\pi}_{\gamma}$ \\
$V$ & $V$ & $V=V$ \\
$\pi_{V}$ & $p_{V}$ & $p_{V}=\left(\pi_{V} / s\right)$ \\
$s$ & $s$ & $s=s$ \\
$d \tau$ & $d t$ & $d t=\frac{d \tau}{s}$ \\
- & $\zeta$ & $\zeta=\frac{1 d s}{s d t}$ \\
$\mathbf{z}_{\gamma}=\mathbf{A}_{\gamma}^{T}\left(V^{1 / 3} \boldsymbol{\rho}_{\gamma}\right) \boldsymbol{\mu}_{\gamma}$ & $\mathbf{z}_{\gamma}=\mathbf{A}_{\gamma}^{T}\left(\mathbf{r}_{\gamma}\right) \boldsymbol{\mu}_{\gamma}$ & $\mathbf{z}_{\gamma}=\mathbf{z}_{\gamma}$ \\
\hline \hline
\end{tabular}

$$
P_{i n s t}^{m o l}=\frac{1}{3 V}\left\{\sum_{\gamma} \frac{\mathbf{P}_{\gamma}^{T} \mathbf{P}_{\gamma}}{M_{\gamma}}+\sum_{\gamma} \mathbf{R}_{\gamma}^{T} \mathbf{F}_{\gamma}\right\}
$$

$\mathbf{F}_{\gamma}$ is the total force on molecule $\gamma$ and $\mathbf{F}_{\gamma}=\sum_{i}^{n} \mathbf{f}_{i, \gamma}$, where $\mathbf{f}_{i, \gamma}$ is the three-dimensional force vector acting on atom $i$ in molecule $\gamma$.

\section{B. Energy dissipation}

Both sets of equations of motion (3)-(7) and (17)-(21) are consistent with the NPT ensemble. However, an efficient simulation scheme should give a quick response to an externally applied perturbation in order to drive the system back to equilibrium. The two simulation schemes presented here involve quite different response mechanisms. While in the atomic scaling method all degrees of freedom are involved in the pressure steering, only the centers of mass respond directly to local stress when the molecular scaling method is used. To quantify these differences we consider appropriate response functions in the framework of linear response theory [14].

\section{Hamiltonians}

In order to relate the rate of energy or heat dissipation to mechanical quantities, we start with the corresponding NoséAndersen Hamiltonians $\mathcal{H}_{v}^{\text {atm }}$ and $\mathcal{H}_{v}^{\text {mol }}$ in terms of virtual variables, since these virtual variables involve no implicit volume dependence:

$$
\begin{aligned}
\mathcal{H}_{v}^{a t m}= & \sum_{\gamma} \frac{\boldsymbol{\pi}_{\gamma}^{T} \mathbf{M}_{\gamma}^{-1} \boldsymbol{\pi}_{\gamma}}{2 V^{2 / 3} s^{2}}+\frac{\pi_{V}^{2}}{2 s^{2} W_{V}}+\frac{\pi_{s}^{2}}{2 W_{s}}+\mathcal{V}\left(\left\{V^{1 / 3} \boldsymbol{\rho}_{\gamma}\right\}\right) \\
& +P_{e x t} V+(f+1) k_{B} T \ln s \\
\mathcal{H}_{v}^{m o l}= & \sum_{\gamma}\left\{\frac{\tilde{\boldsymbol{\pi}}_{\gamma}^{T} \mathbf{M}_{\gamma}^{-1} \tilde{\boldsymbol{\pi}}_{\gamma}}{2 s^{2}}+\frac{\boldsymbol{\Pi}_{\gamma}^{T} \boldsymbol{\Pi}_{\gamma}}{M_{\gamma} 2 s^{2} V^{2 / 3}}\right\}+\frac{\pi_{V}^{2}}{2 s^{2} W_{V}}+\frac{\pi_{s}^{2}}{2 W_{s}} \\
& +\mathcal{V}\left(\left\{\tilde{\boldsymbol{\rho}}_{\gamma}+V^{1 / 3} \mathbf{I} \mathcal{R}_{\gamma}\right\}\right)+P_{e x t} V+(f+1) k_{B} T \ln s .
\end{aligned}
$$

$\mathcal{V}$ stands for the potential energy, $\pi_{V}$ is the virtual momentum associated with the volume, and $s$ and $\pi_{s}$ are the Nosé variable and its conjugate momentum, respectively. The virtual Hamiltonian $\mathcal{H}_{v}^{a t m}$ corresponding to the atomic scaling procedure depends on $\boldsymbol{\rho}_{\gamma}, \boldsymbol{\pi}_{\gamma}$, which are the virtual coordinates and conjugate momenta, respectively. In contrast, the virtual Hamiltonian $\mathcal{H}_{v}^{\text {mol }}$ is formulated in terms of the $3 n$-dimensional vectors $\tilde{\boldsymbol{\rho}}_{\gamma}$ and the three-dimensional vectors $\mathcal{R}_{\gamma}$, which are the virtual variables associated with relative and center-of-mass coordinates, respectively. The corresponding momenta are denoted as $\tilde{\boldsymbol{\pi}}_{\gamma}$ and $\boldsymbol{\Pi}_{\gamma}$. The scaling rules that connect these virtual variables with the physical (real) coordinates and momenta are listed in Tables I and II.

From the above Hamiltonians and the constraints the virtual equations of motion can be derived. To obtain the equations of motion (3)-(7) and (17)-(21) the respective scaling rules are to be applied; this includes Nosé's time scaling procedure (Tables I and II). As outlined in [3], in the case of

TABLE II. Molecular scaling.

\begin{tabular}{ccc}
\hline \hline Virtual variable & Real variable & Connection \\
\hline$\tilde{\boldsymbol{\rho}}_{\gamma}$ & $\tilde{\mathbf{r}}_{\gamma}$ & $\tilde{\mathbf{r}}_{\gamma}=\tilde{\boldsymbol{\rho}}_{\gamma}$ \\
$\mathcal{R}_{\gamma}$ & $\mathbf{R}_{\gamma}$ & $\mathbf{R}_{\gamma}=V^{+1 / 3} \mathcal{R}_{\gamma}$ \\
$\tilde{\boldsymbol{\pi}}_{\gamma}$ & $\tilde{\mathbf{p}}_{\gamma}$ & $\mathbf{r}_{\gamma}=\tilde{\boldsymbol{\rho}}_{\gamma}+V^{1 / 3} \mathbf{I} \mathcal{R}_{\gamma}$ \\
$\boldsymbol{\Pi}_{\gamma}$ & $\mathbf{P}_{\gamma} \tilde{\mathbf{p}}_{\gamma}=s^{-1} \tilde{\boldsymbol{\pi}}_{\gamma}$ \\
$V$ & & $\mathbf{P}_{\gamma}=V^{-1 / 3} s^{-1} \mathbf{\Pi}_{\gamma}$ \\
$\pi_{V}$ & $V$ & $\left.s^{-1} \tilde{\boldsymbol{\pi}}_{\gamma}+\mathbf{M}_{\gamma} \mathbf{I I I}_{\gamma} / M_{\gamma} V^{1 / 3}\right\}$ \\
$s$ & $p_{V}$ & $\mathbf{p}_{\gamma}=V$ \\
$d \tau$ & $s$ & $p_{V}=\left(\pi_{V} / s\right)$ \\
& $d t$ & $s=s$ \\
$\mathbf{z}_{\gamma, v}=\mathbf{A}_{\gamma}^{T}(\tilde{\boldsymbol{\rho}}) \tilde{\boldsymbol{\mu}}_{\gamma}+\mathbf{M}_{\gamma} \mathbf{I} \boldsymbol{\xi}_{\gamma}$ & $\zeta$ & $d t=\frac{d \tau}{s}$ \\
& $\mathbf{z}_{\gamma}=\mathbf{A}_{\gamma}^{T}\left(\tilde{\mathbf{r}}_{\gamma}\right) \tilde{\boldsymbol{\mu}}_{\gamma}=\mathbf{A}_{\gamma}^{T}\left(\mathbf{r}_{\gamma}\right) \tilde{\boldsymbol{\mu}}_{\gamma}$ & $\zeta=\frac{1 d s}{s d t}$ \\
\hline \hline
\end{tabular}


the atomic scaling procedure the proper set of constraints must include the momentum constraints for the virtual momenta

$$
\mathbf{A}_{\gamma} \mathbf{M}_{\gamma}^{-1} \boldsymbol{\pi}_{\gamma}=\mathbf{0}
$$

It follows then from the scaling relations $\mathbf{p}_{\gamma}=s^{-1} V^{-1 / 3} \boldsymbol{\pi}_{\gamma}$ that the real momenta fulfill the proper constraints (12) too. In the case of the molecular scaling procedure it is not necessary to include the momentum constraints explicitly. Since the geometrically intramolecular constraints can always be formulated in terms of the relative coordinates $\tilde{\boldsymbol{\rho}}_{\gamma}$ only and since these relative coordinates do not interfere with the length scaling $\left(\tilde{\boldsymbol{\rho}}_{\gamma}=\tilde{\boldsymbol{r}}_{\gamma}\right.$, where $\tilde{\boldsymbol{r}}_{\gamma}$ are the real positions relative to the center of mass of molecule $\gamma$; compare Table II), the momentum constraints are automatically fulfilled and in this sense redundant in the molecular scaling procedure. However, the virtual variables corresponding to the relative coordinates are not independent variables because they obey the equation

$$
\sum_{i}^{n} m_{i} \tilde{\boldsymbol{\rho}}_{i, \gamma}=\mathbf{0}
$$

where $\tilde{\boldsymbol{\rho}}_{i, \gamma}$ is the virtual three-dimensional position vector of atom $i$ in molecule $\gamma$ relative to the center of mass. The conditions (27) have to be considered as extra constraints when the virtual equations of motion are to be derived from Eq. (25) [7].

Collecting the respective virtual variables in the set $\left\{q^{\nu}\right\}$ and all corresponding momenta in the set $\left\{p_{\nu}\right\}$, where either $\nu=1, \ldots, 3 N+2$ (atomic scaling) or $\nu=1, \ldots, 3 N+3 K$ +2 (molecular scaling), Hamiltonian equations for the respective virtual variables can be derived from the variational principle

$$
\delta S=\delta \int_{\tau_{0}}^{\tau_{1}} d \tau\left(\sum_{\nu} \dot{q}^{\nu} p_{\nu}-\mathcal{H}_{v}\right)=0
$$

where the velocities are to be expressed in terms of coordinates and momenta. Due to the respective set of constraints, the variations appearing in Eq. (28) are not independent. In order to take the constraints into account the new Hamiltonians $\mathcal{H}_{v}^{a t m, *}$ and $\mathcal{H}_{v}^{\text {mol,* }}$ can be introduced [3]

$\mathcal{H}_{v}^{a t m, *}=\mathcal{H}_{v}^{a t m}-\sum_{\gamma}^{K} \boldsymbol{\sigma}_{\gamma}^{T}\left(V^{1 / 3} \boldsymbol{\rho}_{\gamma}\right) \boldsymbol{\mu}_{\gamma}+\sum_{\gamma}^{K} \boldsymbol{\pi}_{\gamma}^{T} \mathbf{A}_{\gamma}^{T}\left(V^{1 / 3} \boldsymbol{\rho}_{\gamma}\right) \boldsymbol{\kappa}_{\gamma}$,

$\mathcal{H}_{v}^{m o l, *}=\mathcal{H}_{v}^{m o l}-\sum_{\gamma}^{K} \boldsymbol{\sigma}_{\gamma}^{T}\left(\tilde{\boldsymbol{\rho}}_{\gamma}\right) \tilde{\boldsymbol{\mu}}_{\gamma}-\sum_{\gamma}^{K} \boldsymbol{\xi}_{\gamma}^{T} \sum_{i}^{n} m_{i} \tilde{\boldsymbol{\rho}}_{i, \gamma}$.

Here we introduced the $n_{c}$-dimensional vectors $\boldsymbol{\sigma}_{\gamma}$ $=\left(\sigma_{\gamma}^{1}, \ldots, \sigma_{\gamma}^{n^{c}}\right)^{T}$. The Lagrangian multipliers $\boldsymbol{\mu}_{\gamma}$ $=\left(\mu_{1, \gamma}, \ldots, \mu_{n_{c}, \gamma}\right)^{T}, \quad \boldsymbol{\kappa}_{\gamma}=\left(\kappa_{1, \gamma}, \ldots, \kappa_{n_{c}, \gamma}\right)^{T}$, and $\tilde{\boldsymbol{\mu}}_{\gamma}$ $=\left(\tilde{\mu}_{1, \gamma}, \ldots, \tilde{\mu}_{n_{c}, \gamma}\right)^{T}$ and the three-dimensional $\boldsymbol{\xi}_{\gamma}$ appear now as independent new variables. Inserting the Hamiltonians (29) and (30) into the action integral (28) allows one to consider an unconstrained variational problem. The virtual equations of motion are then obtained in the standard forms $\dot{q}^{\nu}=\partial \mathcal{H}_{v}^{*} / \partial p_{\nu}$ and $\dot{p}_{\nu}=-\partial \mathcal{H}_{v}^{*} / \partial q^{\nu}$ and the Lagrangian multipliers are to be determined by the requirement that the respective constraints are fulfilled.

\section{Response theory}

Since the virtual Hamiltonians (29) and (30) are formulated in terms of the virtual variables that involve no implicit volume dependence, they are suitable to construct the proper perturbation Hamiltonians corresponding to an externally applied compression or dilatation. To describe such a perturbation we replace in the Hamiltonians $\mathcal{H}_{v}^{a t m, *}$ and $\mathcal{H}_{v}^{\text {mol,**}}$ the volume $V$ by its perturbed value $V^{\prime}$,

$$
V \rightarrow V^{\prime}=[1+\tilde{\alpha}(\tau)] V,
$$

where the relative compression factor $\tilde{\alpha}(\tau)=\left(V^{\prime}-V\right) / V$ depends on virtual time. Since we are interested in small perturbations $|\tilde{\alpha}(\tau)| \ll 1$, the corresponding perturbations of the Hamiltonians $\Delta \mathcal{H}_{v}^{a t m, *}(\tau)$ and $\Delta \mathcal{H}_{v}^{m o l}, *(\tau)$ can be obtained from the expressions (29) and (30) by linearization with respect to $\tilde{\alpha}$ :

$$
\mathcal{H}_{v, \text { pert }}^{*}(\tau)=\mathcal{H}_{v}^{*}+\underbrace{\tilde{\alpha}(\tau) V \frac{\partial \mathcal{H}_{v}^{*}}{\partial V}}_{\Delta \mathcal{H}_{v}^{*}}+\mathcal{O}\left(\tilde{\alpha}^{2}\right)
$$

When the respective connections between virtual and real variables listed in Tables I and II are applied, the perturbations $\Delta \mathcal{H}^{a t m, *}$ and $\Delta \mathcal{H}^{m o l, *}$ can concisely be expressed in terms of the real variables

$$
\begin{aligned}
& \Delta \mathcal{H}^{a t m, *}=-\alpha(t)\left(P_{\text {inst }}^{a t m}-P_{\text {ext }}\right) V, \\
& \Delta \mathcal{H}^{m o l, *}=-\alpha(t)\left(P_{\text {inst }}^{\text {mol }}-P_{\text {ext }}\right) V .
\end{aligned}
$$

Here the definitions of the instantaneous pressures (16) and (23) in terms of the real variables were used and $\alpha(t)$ $=\tilde{\alpha}(\tau(t))$ may now be considered as a function of real time. Introducing the abbreviation $A=\left(P_{\text {inst }}^{a t m / m o l}-P_{\text {ext }}\right) V$, the respective perturbation terms can be written as $\Delta \mathcal{H}^{*}=$ $-\alpha(t) A$. For any function of the real variables $B$, the deviation due to the compression from its equilibrium value $\langle B\rangle_{e q}$ in the unperturbed ensemble $\langle\delta B\rangle(t) \equiv\langle B\rangle(t)-\langle B\rangle_{e q}$ can be expressed as

$$
\langle\delta B\rangle(t)=\int_{-\infty}^{t} d t^{\prime} \Phi_{B A}\left(t-t^{\prime}\right) \alpha\left(t^{\prime}\right)+O\left(\alpha^{2}\right),
$$

where $\Phi_{B A}$ is the linear response function. Evans and Holian have proved that the standard Kubo form [14] for the linear response function $\Phi_{B A}$ still holds, even when the equations of motion are of Nosé-Hoover type $[17,18]$. Therefore, we use the standard Kubo form of the linear response function too. According to standard linear response theory [14], the 
time-dependent linear response functions $\Phi_{B A}$ are given as time correlation functions, which are to be evaluated in the unperturbed ensemble

$$
\Phi_{B A}(t)=-\frac{1}{k_{B} T}\langle A(0) \dot{B}(t)\rangle_{e q} .
$$

The difference in the response mechanisms for the atomic and the molecular scaling procedure rests on the different perturbation variables, either $A^{a t m}=\left(P_{\text {inst }}^{a t m}-P_{e x t}\right) V$ or $A^{m o l}$ $=\left(P_{\text {inst }}^{m o l}-P_{\text {ext }}\right) V$.

Having established the general form of the perturbation corresponding to an externally applied compression or dilatation (33) and (34), we follow Kubo [14] in order to discuss how the work done by the external perturbation can be dissipated as heat into the environment. Thermodynamically, the average rate of enthalpy loss $-\partial\left(E+P_{e x t} V\right) / \partial t$ equals the rate of heat dissipation $\partial Q / \partial t$. Assuming for simplicity a periodic (monochromatic) compression factor $\alpha(t)$ $=\operatorname{Re}\left\{X_{0} \exp \left(-i \omega_{0} t\right)\right\}$, the average rate of heat dissipation over a cycle period $2 \pi / \omega_{0}$ can be written as

$$
\begin{aligned}
\frac{\partial Q}{\partial t} & =-\overline{\dot{\alpha} \int_{0}^{\infty} d t^{\prime} \Phi_{A A}\left(t^{\prime}\right) \alpha\left(t-t^{\prime}\right)} \\
& =-\overline{\frac{\alpha}{2}\left[X_{0} e^{-i \omega_{0} t} \chi_{A A}\left(\omega_{0}\right)+X_{0}^{*} e^{i \omega_{0} t} \chi_{A A}^{*}\left(\omega_{0}\right)\right]} .
\end{aligned}
$$

Here $\chi_{A A}(\omega)=\int_{0}^{\infty} d t e^{i \omega t} \Phi_{A A}(t)$ denotes the Fourier-Laplace transform of the response function $\Phi_{A A}$ describing the response mechanism of $A$ itself. The asterisk in Eq. (37) denotes the complex conjugate and the overbar stands for a time average over the period $2 \pi / \omega_{0}$. Inserting in this expression $\dot{\alpha}(t)=i \omega_{0} / 2\left[-X_{0} \exp \left(-i \omega_{0} t\right)+X_{0}^{*} \exp \left(i \omega_{0} t\right)\right]$ and regarding that $\overline{\exp \left( \pm i 2 \omega_{0} t\right)}=0$ yields

$$
\dot{Q}=\left|X_{0}\right|^{2} \frac{\omega_{0}}{2} \operatorname{Im}\left\{\chi_{A A}\left(\omega_{0}\right)\right\} .
$$

Finally, the imaginary part of the complex susceptibility $\operatorname{Im}\left\{\chi_{A A}(\omega)\right\}$ can be reformulated as

$$
\operatorname{Im}\left\{\chi_{A A}(\omega)\right\}=\int_{0}^{\infty} d t \sin (\omega t) \Phi_{A A}(t)=\frac{\omega}{2 k_{B} T} \underbrace{\int_{-\infty}^{\infty} d t \cos (\omega t)\langle A(0) A(t)\rangle_{e q}}_{I_{\{A\}}(\omega)}
$$

This shows that the average rate of heat dissipation is proportional to $\omega_{0}^{2} I\left(\omega_{0}\right)$. Here $I_{\{A\}}(\omega)$ denotes the power spectrum of $A$, given as the Fourier transform of the autocorrelation function of $A$. In the following $\omega$ is understood as the frequency of compression $\omega \equiv \omega_{0}$. Thus the heat dissipation spectrum can be computed from the autocorrelation function of the respective property $A$. The shape of the heat dissipation spectrum indicates the frequency range contributing to the dissipation of stress as a consequence of externally applied compression or dilatation. Summarizing, we can say that in the case of atomic scaling the heat dissipation spectrum $\dot{Q}^{a t m}(\omega)$ corresponding to an externally applied compression is given by

$$
\dot{Q}^{a t m}(\omega) \propto \omega^{2} I_{\left\{\left(P_{\text {inst }}^{a t m}-P_{\text {ext }}\right) V\right\}}(\omega),
$$

whereas $\dot{Q}^{\text {mol }}(\omega)$ reads for molecular scaling

$$
\dot{Q}^{m o l}(\omega) \propto \omega^{2} I_{\left\{\left(P_{i n s t}^{m o l}-P_{e x t}\right) V\right\}}(\omega) .
$$

\section{MATERIALS AND METHODS}

\section{Molecular dynamics simulation}

Two different systems of $n$-alkane chains, each containing 2560 beads, have been simulated with the atomic and molecular pressure baths described above (four simulations in total). In all NPT simulations the external temperature and pressure were fixed to $T=303 \mathrm{~K}$ and $P_{\text {ext }}=1 \mathrm{~atm}$, respectively. The total momentum of the two systems was always zero and all bond lengths were constrained. The first system consists of $256 n$-decane molecules ("short chains") corresponding to $f=5373$ degrees of freedom. The second system ("long chains") consists of $80 n$-alkanes, each containing 32 carbons ( $n$-dotriacontanes). This system corresponds to $f$ $=5197$ degrees of freedom. Both systems were prepared in a nonequilibrium start configuration to observe relaxation into equilibrium. For the NPT simulations of the decanes we started with an existing isotropic configuration of 256 molecules that was then equilibrated in an $N V T$ simulation at temperature $T=522 \mathrm{~K}$ and a box volume $V=97.77 \mathrm{~nm}^{3}$. Before starting the isobaric simulations (atomic scaling and molecular scaling) we rescaled all velocities such that the initial temperature was $T=303 \mathrm{~K}$, keeping the volume for the hightemperature configuration. In view of the longer relaxation times to be expected for the long chains we prepared a configuration supposedly somewhat closer to equilibrium. First we constructed an "all-trans" configuration for the 80 chains. Then we heated the system again to $T=522 \mathrm{~K}$, however adjusting the box volume to a value of $V=67.28 \mathrm{~nm}^{3}$. The heating phase was followed by an NVT simulation of 100 ps at $T=303 \mathrm{~K}$, keeping the same volume, before starting the NPT simulations. The value of $67.28 \mathrm{~nm}^{3}$ for the volume is an estimate that was obtained from Fig. 1 in [19] by extrapolating to a chain length 32 . The simulations for the different pressure baths started with exactly the same initial configuration for the decanes and the dotriacontane chains. Both systems were simulated with the same force field and simulation parameters. A time step of $2 \mathrm{fs}$ was used for integrating the equations of motion. The relaxation time $\tau_{T}$ 


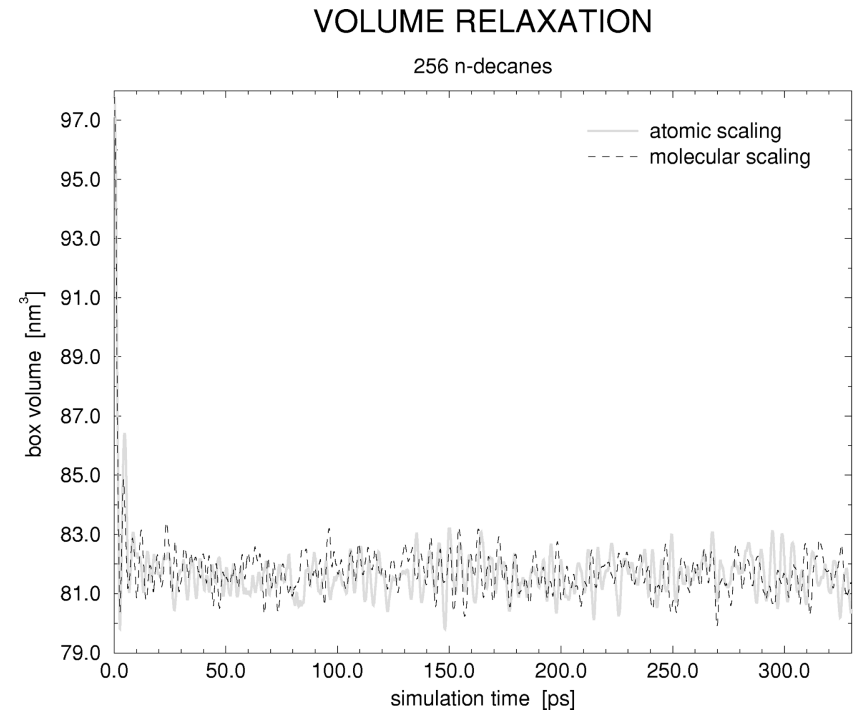

FIG. 1. Relaxation of the box volume of $256 n$-decane molecules as a function of simulation time. The gray solid curve corresponds to atomic scaling, the black dashed curve corresponds to molecular scaling.

determining the "Nosé mass" $W_{S}=(f+1) k_{B} T \tau_{T}^{2}$ was chosen to be $\tau_{T}=0.2 \mathrm{ps}$. Andersen's piston mass having the physical dimension $\mathrm{kg} / \mathrm{m}^{4}$ can also be formulated in terms of a relaxation time $W_{V}=(f+1) k_{B} T\left[\tau_{P}^{2} / V^{2}(0)\right]$, where $V(0)$ is the initial volume. We fixed $W_{V}$ by choosing $\tau_{P}=1.6 \mathrm{ps}$ in all simulations. The equations of motion were integrated with a modified velocity Verlet integrator. The algorithm for atomic scaling is described in detail in the Appendix. We used ten iterations each step for the iterative process (vii) described in the Appendix. Possibly fewer iterations could also be used. For the molecular scaling method the velocitydependent acceleration terms in the equations of motion have also been treated iteratively, very similarly to the scheme for the atomic scaling described in the Appendix. The bond length constraints were considered to be fulfilled for errors

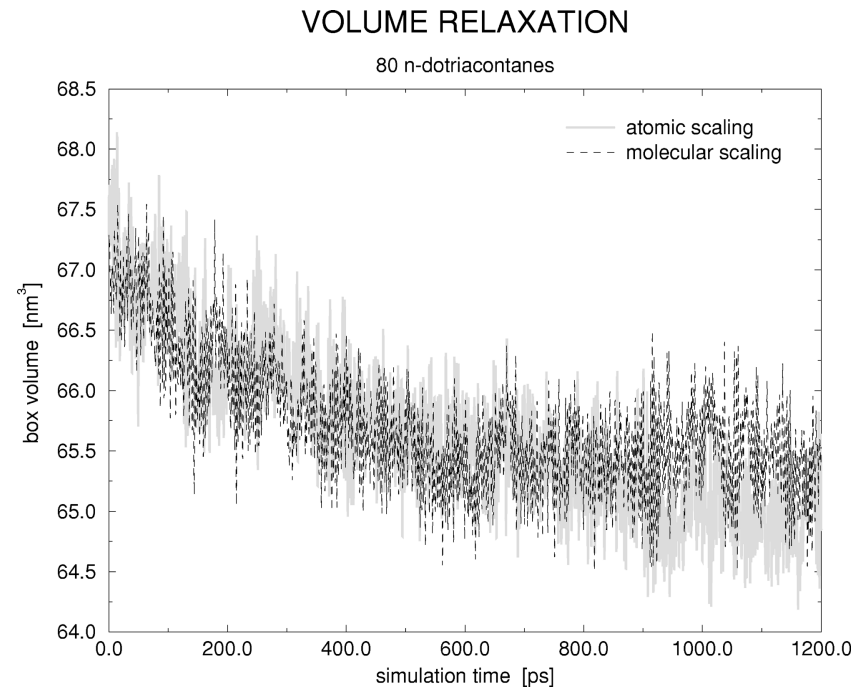

FIG. 2. Relaxation of the box volume of $80 n$-dotriacontane molecules as a function of simulation time. The gray solid curve corresponds to atomic scaling, the black dashed curve corresponds to molecular scaling.

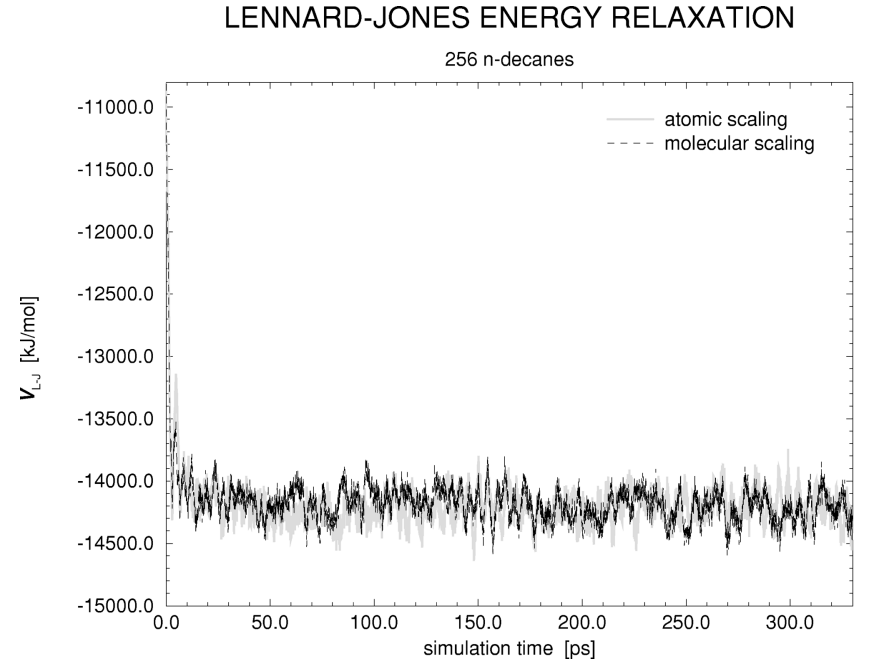

FIG. 3. Relaxation of the Lennard-Jones energy of $256 n$-decane molecules as a function of simulation time. The gray solid curve corresponds to atomic scaling, the black dashed curve corresponds to molecular scaling.

smaller than a relative tolerance of $10^{-8}$. Except for the torsion potential, the force field was Toxvaerd's united atom force field for decanes described in [19]. The torsion potential was that of Smith and Jaffe [20], which has been expanded into a polynomial in $\cos \left(\Psi_{\text {torsion }}\right)$. The coefficients of this expansion can be found in Table I in [21]. The torsion energies reported below were calculated without use of the first coefficient corresponding to $\left[\cos \left(\Psi_{\text {torsion }}\right)\right]^{0}=$ const. This alters neither the forces nor the virial, but causes a shift in the torsion potential. To mimic an infinite system, cubic periodic boundary conditions were applied. Since we applied always the atomic version of periodic boundary conditions [22], special care was taken when the molecular virial was computed. As described in [9], the molecular virial can be computed in a way consistent with (atomic) periodic boundaries as $\sum_{\gamma} \mathbf{R}_{\gamma} \cdot \mathbf{F}_{\gamma}=\sum_{i \gamma<j \delta}^{\prime}\left(\mathbf{r}_{i \gamma, j \delta}^{N I}-\tilde{\mathbf{r}}_{i, \gamma}+\tilde{\mathbf{r}}_{j, \delta}\right) \cdot \mathbf{f}_{i \gamma, j \delta}$. The summation on the right-hand side runs over all pairs of at-

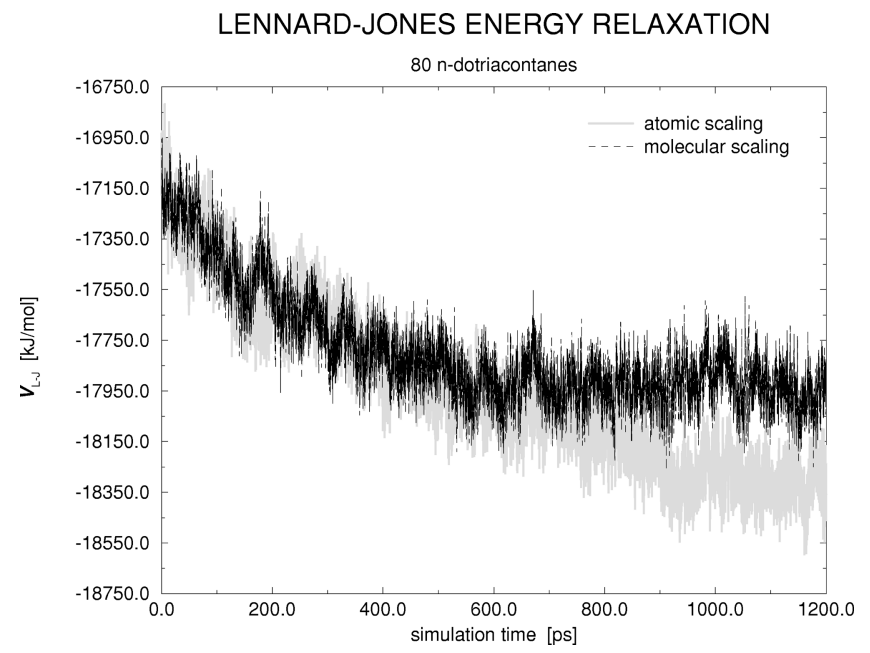

FIG. 4. Relaxation of the Lennard-Jones energy of $80 n$ dotriacontane molecules as a function of simulation time. The gray solid curve corresponds to atomic scaling, the black dashed curve corresponds to molecular scaling. 
TORSION and BENDING ANGLE ENERGY RELAXATION

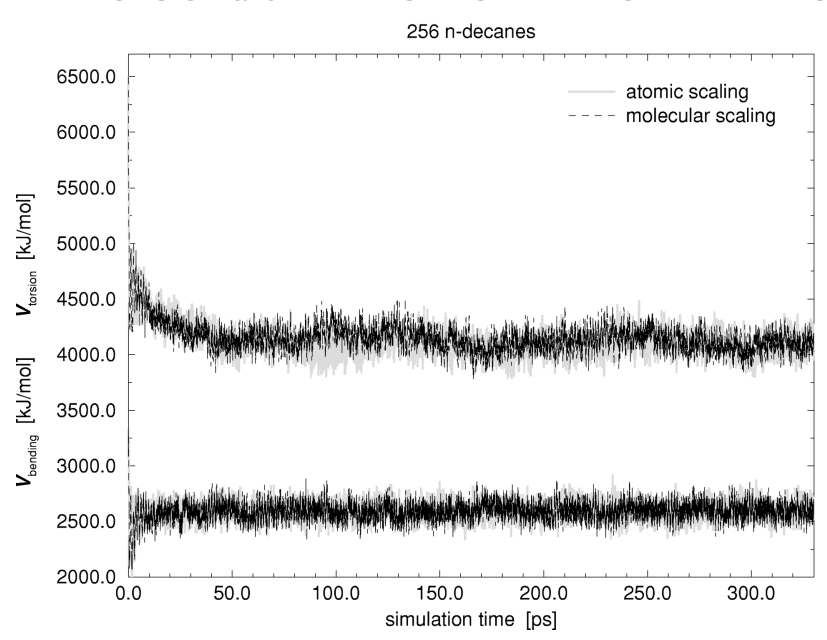

FIG. 5. Relaxation of the torsion energy (upper curves) and bending angle energy (lower curves) of $256 n$-decane molecules as a function of simulation time. The gray solid curves correspond to atomic scaling, the black dashed curves correspond to molecular scaling.

oms for which atom $i$ and atom $j$ are in different molecules $(\gamma \neq \delta)$ and the superscript NI denotes the three-dimensional nearest image distance vector $\mathbf{r}_{i \gamma, j \delta}^{N I}$ between atoms $i \gamma$ and $j \delta$, while $\tilde{\mathbf{r}}_{i \gamma}$ is the three-dimensional position vector of atom $i$ in molecule $\gamma$ relative to the center of mass of molecule $\gamma$. No long-range corrections, for either the pressure or the energies were taken into account; instead we used a relatively large cutoff distance of $1.6 \mathrm{~nm}$, which corresponds to more than $4 \sigma$, where $\sigma$ denotes the length diameter in the Lennard-Jones potential. This is considered to be a reasonably accurate approximation for nonpolar systems [23]. The Verlet neighbor list technique was applied, using a shell radius of $1.969 \mathrm{~nm}$ for all beads in the pair list, which was updated every ten simulation steps. The system of decanes was simulated for $330 \mathrm{ps}$, using the last $163.84 \mathrm{ps}$ for the

\section{TORSION and BENDING ANGLE ENERGY RELAXATION}

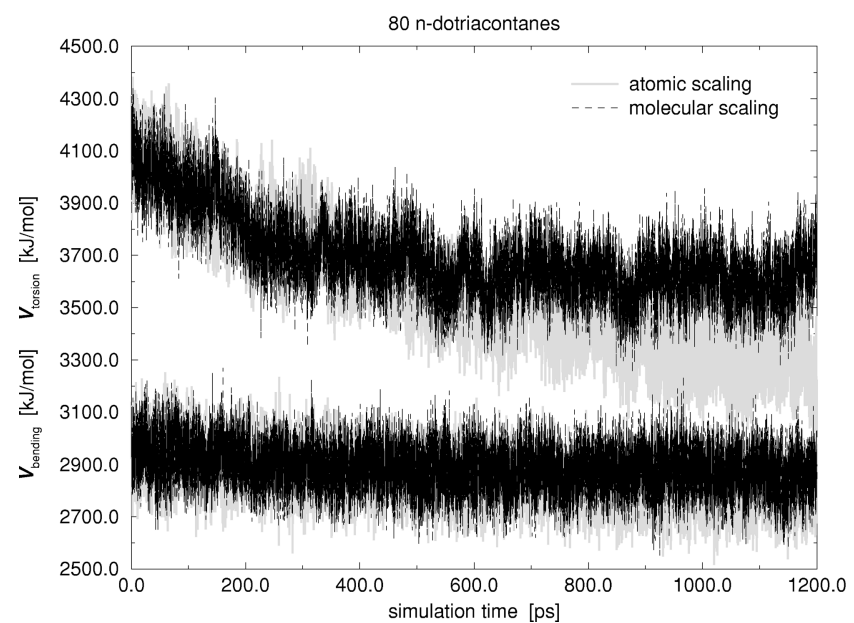

FIG. 6. Relaxation of the torsion energy (upper curves) and bending angle energy (lower curves) of $80 n$-dotriacontane molecules as a function of simulation time. The gray solid curves correspond to atomic scaling, the black dashed curves correspond to molecular scaling.
TABLE III. Averages for $256 n$-decane molecules $\left(\mathrm{C}_{10} \mathrm{H}_{22}\right)$ at $T=303 \mathrm{~K}$ and $P_{\text {ext }}=1$ atm.

\begin{tabular}{lcc}
\hline \hline \multicolumn{1}{c}{ Averaged quantity } & Atomic scaling & Molecular scaling \\
\hline volume $V\left(\mathrm{~nm}^{3}\right)$ & $81.6 \pm 0.58$ & $81.6 \pm 0.56$ \\
$\mathcal{V}_{L J}(\mathrm{~kJ} / \mathrm{mol})$ & $-14212.6 \pm 114.4$ & $-14220.6 \pm 115.7$ \\
$\mathcal{V}_{\text {torsion }}(\mathrm{kJ} / \mathrm{mol})$ & $4090.9 \pm 95.3$ & $4110.9 \pm 92.8$ \\
$\mathcal{V}_{\text {bending }}(\mathrm{kJ} / \mathrm{mol})$ & $2598.6 \pm 73.2$ & $2601.5 \pm 72.9$ \\
enthalpy & & \\
$\quad E+P_{\text {ext }} V(\mathrm{~kJ} / \mathrm{mol})$ & $-746.7 \pm 228.3$ & $-731.3 \pm 210.9$ \\
\hline \hline
\end{tabular}

analysis of equilibrium properties (averages, power spectra, and energy dissipation spectra). This time interval corresponds to $2^{13}=8192$ data points written out every 20 fs and thus enabled us to use the fast Fourier transform technique for the computation of the spectra discussed in Sec. II B. For the longer $n$-dotriacontane chains (32 beads) the system needed a much longer equilibration time. The total simulation length (for each pressure bath) here was $1.2 \mathrm{~ns}$. From the last $327.68 \mathrm{ps}$ corresponding to $2^{14}=16384$ data points, we computed all the listed equilibrium properties.

\section{RESULTS AND DISCUSSION}

Figures 1 and 2 show equilibration curves of the simulated box volume as a function of time. While the volume of the short chains (256n-decanes) in Fig. 1 equilibrates during the first tens of picoseconds, the volume of the longer chains (80 n-dotriacontanes) in Fig. 2 needs hundreds of picoseconds to attain a stable limit. Visualization of the structures of the simulated systems shows that the 32 carbon chains are more or less aligned in parallel at $T=303 \mathrm{~K}$ and $P_{\text {ext }}=1$ atm, with an intramolecular zigzag conformation. In contrast, the decanes form an isotropic fluid. This is consistent with the experimental observation [24] that the alkane chains from length 16 on start to form a wax under normal conditions, where the single chains are ordered in parallel lamellae. The following figures show the energy relaxation behavior: The relaxation of the Lennard-Jones energies is shown in Figs. 3 and 4 for the short and long chains, respectively. Similarly to the volume relaxation, the Lennard-Jones energies of the decanes equilibrate much faster. Again, hundreds of picoseconds are needed for the 32-alkanes. In Figs. 5 and 6 we show the relaxations for the torsion and bond angle energies corresponding to Figs. 3 and 4, respectively. In Tables III and IV we list the averages of various quantities of

TABLE IV. Averages for $80 n$-dotriacontanes $\left(\mathrm{C}_{32} \mathrm{H}_{66}\right)$ at $T$ $=303 \mathrm{~K}$ and $P_{\text {ext }}=1 \mathrm{~atm}$.

\begin{tabular}{lcc}
\hline \hline \multicolumn{1}{c}{ Averaged quantity } & Atomic scaling & Molecular scaling \\
\hline volume $V\left(\mathrm{~nm}^{3}\right)$ & $65.01 \pm 0.32$ & $65.47 \pm 0.35$ \\
$\mathcal{V}_{L J}(\mathrm{~kJ} / \mathrm{mol}$ & $-18264.1 \pm 92.6$ & $-17926.5 \pm 92.9$ \\
$\mathcal{V}_{\text {torsion }} \mathrm{kJ} / \mathrm{mol}$ & $3319.0 \pm 92.9$ & $3610.0 \pm 92.4$ \\
$\mathcal{V}_{\text {bending }}(\mathrm{kJ} / \mathrm{mol})$ & $2807.7 \pm 75.5$ & $2868.6 \pm 78.2$ \\
enthalpy & & \\
$\quad E+P_{\text {ext }} V(\mathrm{~kJ} / \mathrm{mol})$ & $-5601.5 \pm 218.1$ & $-4893.2 \pm 214.5$ \\
\hline \hline
\end{tabular}


ATOMIC and MOLECULAR PRESSURES

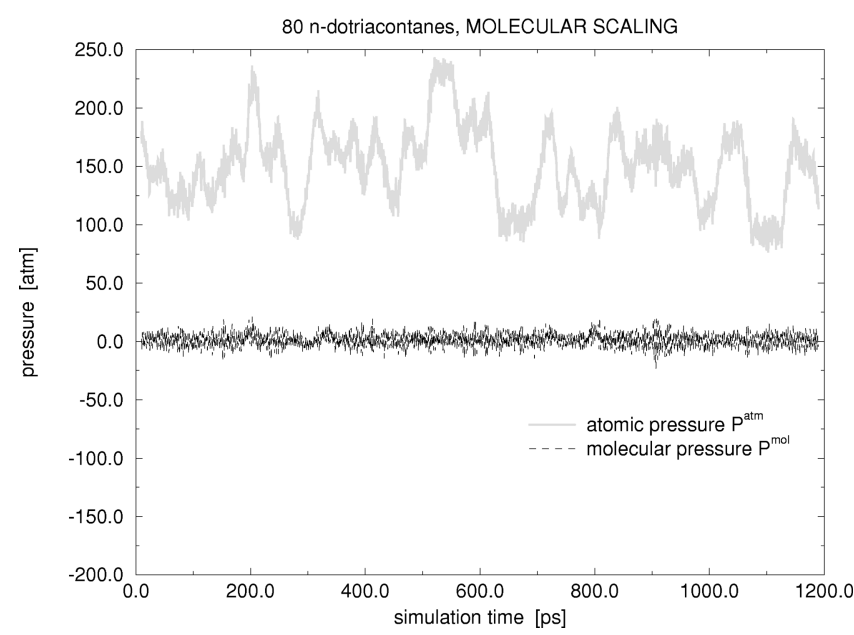

FIG. 7. Molecular scaling for $80 n$-dotriacontane chains: running averages of the atomic pressure (gray solid curve) and the molecular pressure (black dashed curve) as a function of time. Each point in the figure corresponds to an average over 20 ps.

the simulated systems.

As intuitively expected, differences between the relaxation curves and other quantities resulting from using molecular and atomic scaling, respectively, become pronounced for the long chains. It can be seen from the figures that the main differences are between the atomic and the molecular scaling methods in the case of the long 32 carbon $n$-alkanes. There are significant differences in the contributions to the potential energy. Generally, the atomic scaling method produced lower energies on average, which may be considered as an indication for faster relaxation. The total enthalpy difference between the atomic scaling and the molecular scaling method for the 32-alkanes amounts to $708 \mathrm{~kJ} / \mathrm{mol}$, whereas for the decanes this difference is only $15 \mathrm{~kJ} / \mathrm{mol}$. The total enthalpy differences are very close to the sum of the differences in the potential contributions since the kinetic energy

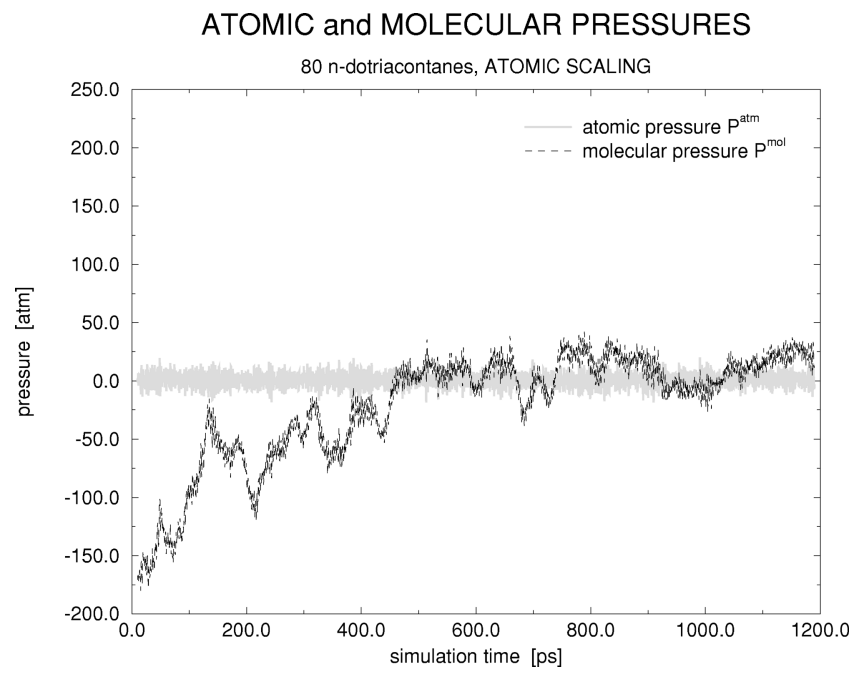

FIG. 8. Atomic scaling for $80 n$-dotriacontane chains: running averages of the atomic pressure (gray solid curve) and the molecular pressure (black dashed curve) as a function of time. Each point in the figure corresponds to an average over 20 ps.

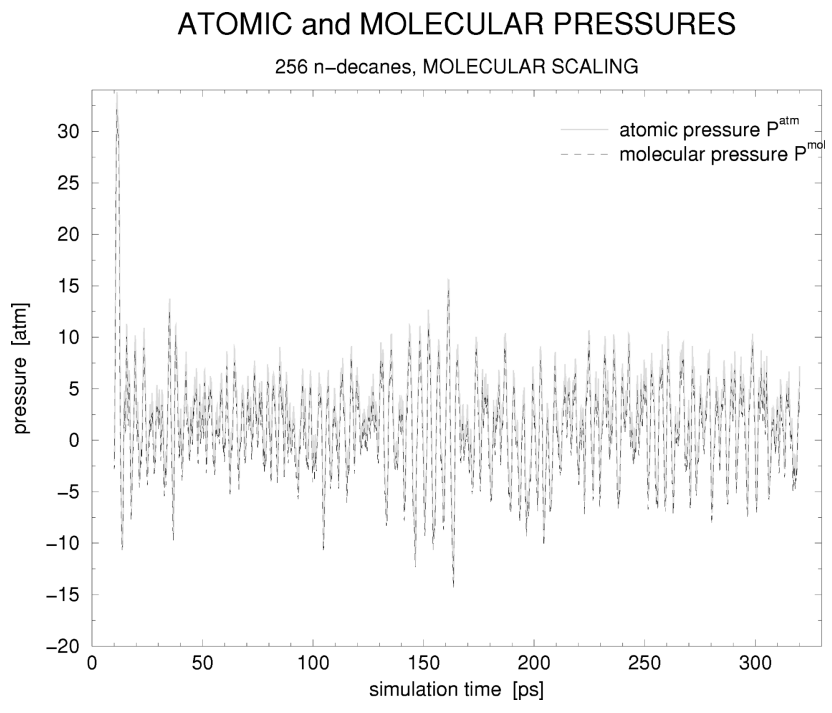

FIG. 9. Molecular scaling for $256 n$-decane chains: running averages of the atomic pressure (gray solid curve) and the molecular pressure (black dashed curve) as a function of time. Each point in the figure corresponds to an average over 20 ps.

differences and the difference in $P_{\text {ext }} V$ are very small compared to the large differences in the potential contributions. An explanation for this large difference in the case of the long 32-bead chains is provided by Figs. 7 and 8. Here, both pressure definitions, the atomic pressure and the molecular pressure, are depicted as running averages during the simulations. Each plotted point corresponds to an average over 20 ps. Such an averaging procedure for obtaining the figures is necessary due to the strong noise in the pressure signal. As can be seen, the molecular scaling method produces atomic pressures that are systematically larger than the controlled molecular pressure. Only the directly controlled molecular pressure [compare the equations of motion (17)-(21)] fluctuates around the prescribed external pressure of $1 \mathrm{~atm}$. Since both pressure definitions must produce equal NPT ensemble averages, it is clear that the molecular scaling method fails to reach the NPT equilibrium distribution, at least in the course of the simulation time (1.2 ns). The situation is different for atomic scaling. Although a long relaxation time (approximately $400 \mathrm{ps}$ ) is needed for convergence of the molecular pressure, both pressures fluctuate finally around $P_{\text {ext }}$. The decanes do not exhibit this behavior at all (see Figs. 9 and 10).

An explanation for these strong differences, in particular for the 32 carbon chains, is provided by the power spectra $I(\omega)$ of $\left(P_{\text {inst }}-P_{\text {ext }}\right) V$ and the corresponding heat dissipation spectra given in Figs. 11-14. Their significance has been discussed in Sec. II B. For both simulated systems, the 256 decanes and the 80 dotriacontane chains, there are pronounced differences at higher frequencies between the atomic and the molecular scaling methods visible in the power spectra (see Figs. 11 and 12). These frequencies (approximately equal to $250 \mathrm{~cm}^{-1}$ for the decanes and approximately equal to $70 \mathrm{~cm}^{-1}$ for the dotriacontanes) correspond to intramolecular modes. The fact that these frequencies are clearly visible in the power spectra $I(\omega)$ corresponding to atomic scaling and practically absent in the corresponding spectrum for molecular scaling shows that the atomic 


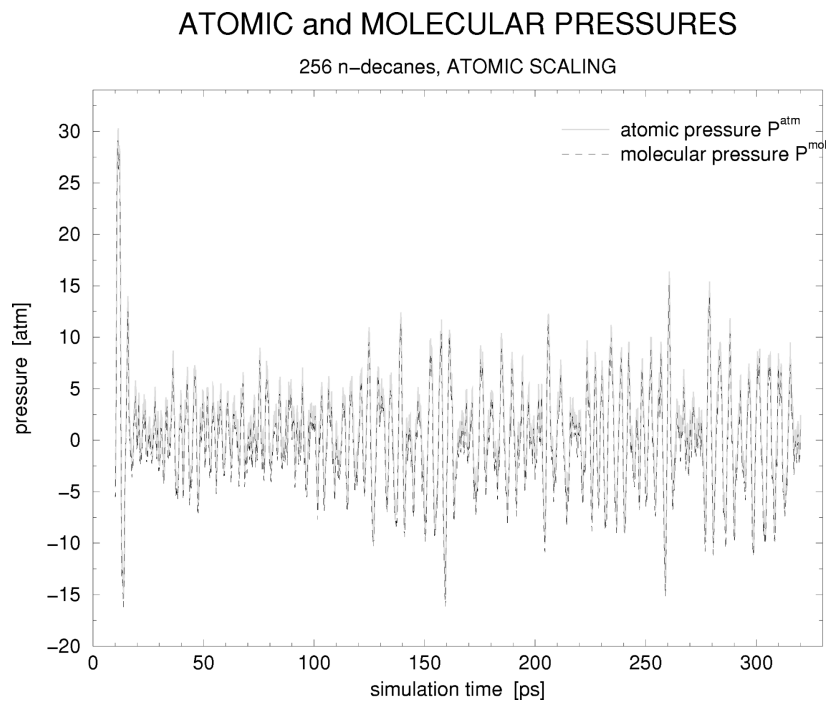

FIG. 10. Atomic scaling for $256 n$-decane chains: Running averages of the atomic pressure (gray solid curve) and the molecular pressure (black dashed curve) as a function of time. Each point in the figure corresponds to an average over 20 ps.

barostat couples to intramolecular motions. A common feature of all power spectra is the large low-frequency peak around wave numbers $7 \mathrm{~cm}^{-1}$ (decanes) and 13 $\mathrm{cm}^{-1}$ (dotriacontanes), no matter which scaling method is applied. The low-frequency peaks can be assigned to the compressibility of the systems of $n$-alkanes, which can be understood as follows [25]. The equation of motion for the volume can be written as $W_{V} \ddot{V} \approx P_{\text {inst }}-P_{\text {ext }}$, when the Nosé thermostating for the piston momentum is neglected. Expanding the volume around its equilibrium value $V_{e q}$ as $V$ $=V_{e q}+\left.(\partial V / \partial P)\right|_{e q}\left(P-P_{e x t}\right)+\cdots$ and solving for $P$ $-P_{\text {ext }}$ shows that the equation of motion for the volume can be formulated approximately as a harmonic oscillator equation

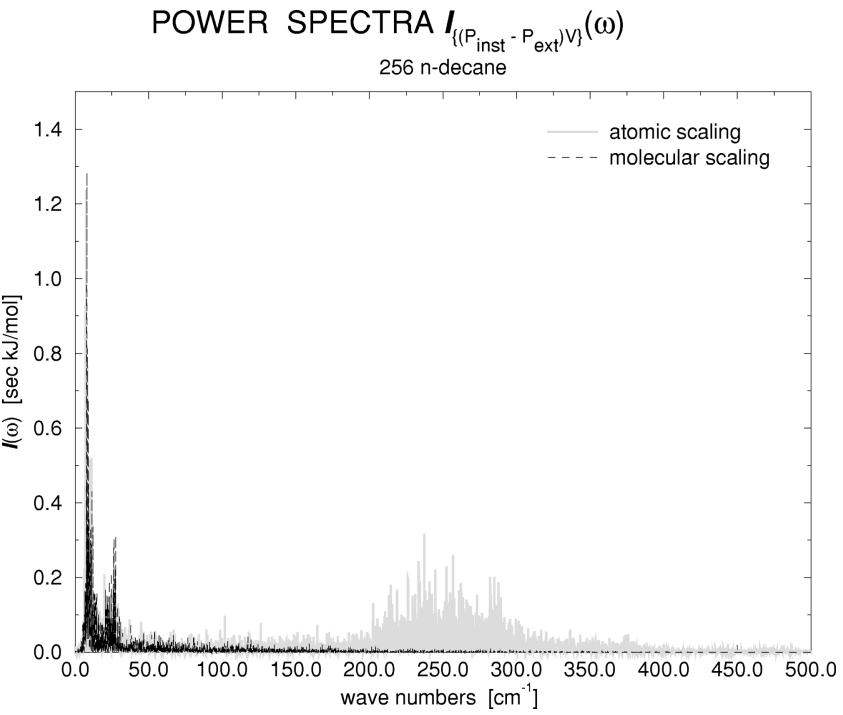

FIG. 11. Power spectra of $\left(P_{\text {inst }}-P_{\text {ext }}\right) V$ obtained from the simulations of $256 n$-decane chains using atomic scaling (gray solid curve) and molecular scaling (black dashed curve).

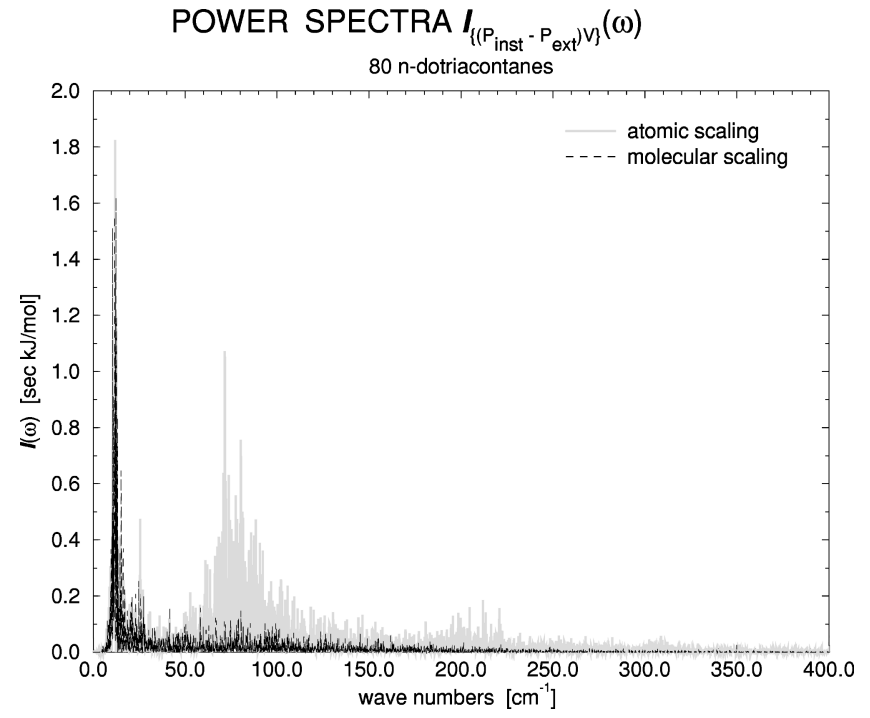

FIG. 12. Power spectra of $\left(P_{\text {inst }}-P_{\text {ext }}\right) V$ obtained from the simulations of $80 n$-dotriacontane chains using atomic scaling (gray solid curve) and molecular scaling (black dashed curve).

$$
\frac{d^{2} V}{d t^{2}} \approx-\frac{1}{\kappa V_{e q} W_{V}}\left(V-V_{e q}\right)
$$

where $\kappa=-\left.(\partial V / \partial P)\right|_{e q} / V_{e q}$ is the (isothermal) compressibility. The frequency of this oscillator is $\omega_{0}=1 / \sqrt{\kappa V_{e q} W_{V}}$. This frequency can also be found in the time evolution of the quantity $A=\left(P_{\text {inst }}-P_{\text {ext }}\right) V$, whose power spectra are shown in Figs. 11 and 12. For the decanes the experimental compressibility is $\kappa=12.34 \times 10^{-10} \mathrm{~Pa}^{-1}$ at $303 \mathrm{~K}$ and 1 atm [26]. Inserting the value for the piston mass, $W_{V}=(f$ $+1) k_{B} T\left[\tau_{P}^{2} / V(0)^{2}\right] \approx 0.602 \times 10^{10} \mathrm{~kg} / \mathrm{m}^{4}$ yields approximately a frequency of $\nu=\omega_{0} / 2 \pi=2.0 \times 10^{11} \mathrm{~s}^{-1}$, corresponding to a wave number of $6.7 \mathrm{~cm}^{-1}$. The large lowfrequency peak (for the atomic scaling as well as for the

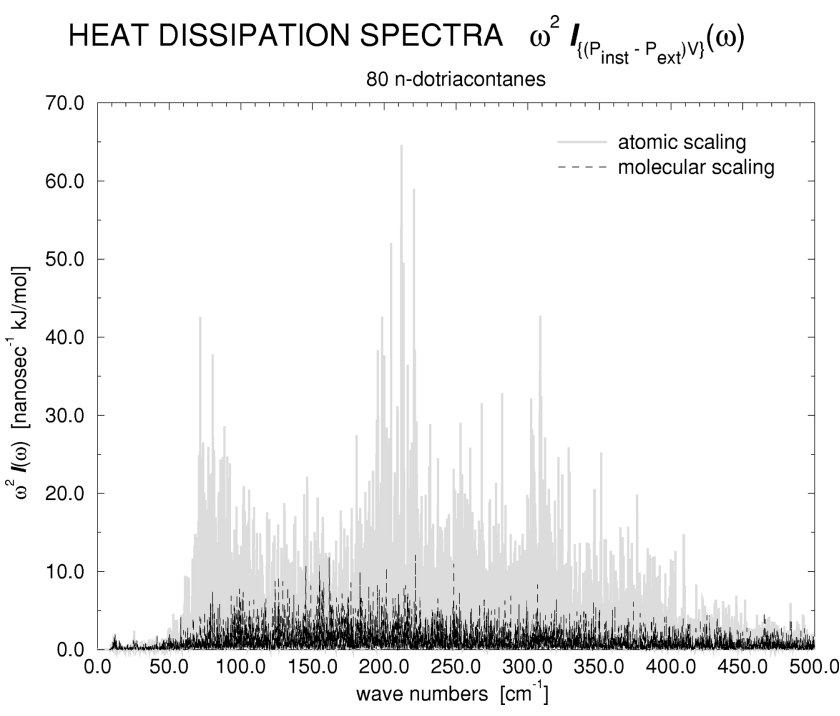

FIG. 13. Heat dissipation spectra $\omega^{2} I_{\left(P_{\text {inst }}-P_{\text {ext }}\right) V}$ corresponding to the power spectra in Fig. 12 obtained from the simulations of 80 $n$-dotriacontane chains using atomic scaling (gray solid curve) and molecular scaling (black dashed curve). 


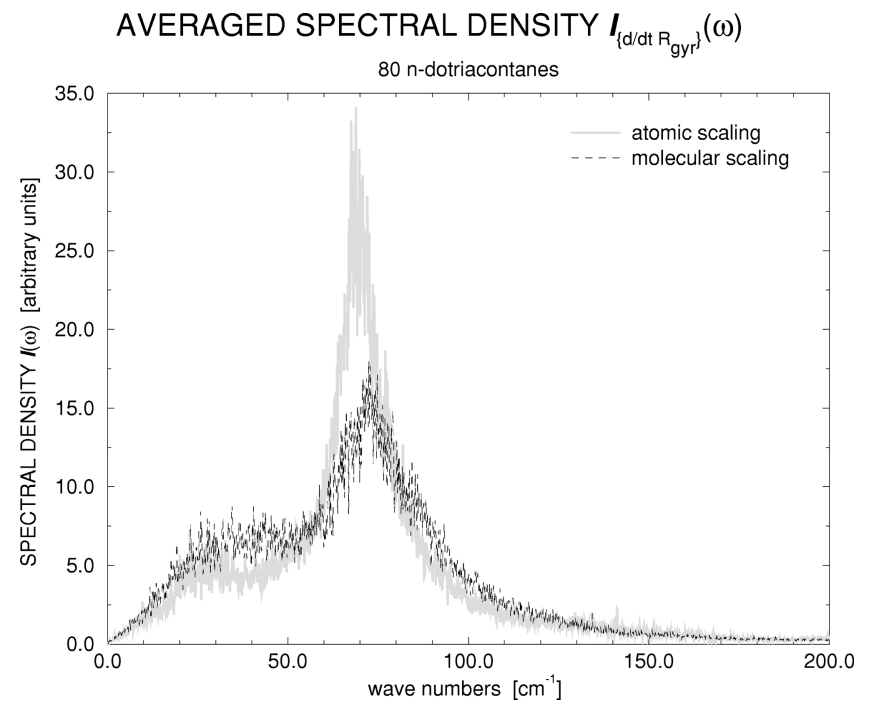

FIG. 14. Averaged power spectra of the time derivative of the radius of gyration of the $80 n$-dotriacontane chains. The averaging is over the 80 power spectra of the single molecules. The gray solid curve corresponds to atomic scaling and the black dashed curve corresponds to molecular scaling.

molecular scaling) in the power spectra in Fig. 11 appears at wave number approximately equal to $7 \mathrm{~cm}^{-1}$, showing that the experimental compressibility is reproduced by the simulations. For the dotriacontanes we found no data for the elastic constants in the literature, but it is plausible that the peak found in the power spectra at approximately $13 \mathrm{~cm}^{-1}$ is related to the elastic constants too. However, these "elastic", low-frequency peaks do not significantly contribute to the rate of energy dissipation since the rate of energy dissipation is proportional to the power spectrum weighted by $\omega^{2}$. Figure 13 shows the "heat dissipation spectra" for the long chains corresponding to the power spectra (Fig. 12), although, at least for the molecular scaling method, no complete equilibrium was established. As can be seen from Fig. 13 , the $(70-80)-\mathrm{cm}^{-1}$ peaks contribute strongly to the rate of stress dissipation in the case of atomic scaling for the long chains. In Ref. [28] these frequencies were assigned to "accordion-like" longitudinal motions of the individual chains performing inelastic neutron scattering experiments on the $n$-dotriacontanes, with the momentum transfer vector oriented parallel and perpendicular to the chain axis. Such motions are responsible for fluctuations of the molecular volume. To confirm that these intramolecular stretching motions are reproduced by the simulations we calculated the power spectra of the time derivatives of the radius of gyration of the molecules. The radius of gyration of molecule $\gamma$ is given by

$$
R_{g y r, \gamma}=\sqrt{\tilde{\mathbf{r}}_{\gamma}^{T} \tilde{\mathbf{r}}_{\gamma}}
$$

where $\tilde{\mathbf{r}}_{\gamma}$ collects all the $3 n$ relative coordinates of molecule $\gamma$ with respect to its center of mass. Thus the corresponding time derivative reads $\dot{R}_{g y r, \gamma}=\hat{\mathbf{e}}_{R_{g y r}}^{T} \dot{\overrightarrow{\mathbf{r}}}_{\gamma}$, where $\hat{\mathbf{e}}_{R_{g y r}}$ $=\tilde{\mathbf{r}}_{\gamma} / R_{g y r, \gamma}$ is the $3 n$-dimensional unit vector in radial direction. The power spectrum of $\dot{R}_{g y r, \gamma}$ is a plausible measure for the contribution of the intramolecular "breathing motions", to the power spectrum obtained from the (Cartesian) velocity autocorrelation function, which is observed by neutron scattering. The spectral densities (averaged over all $80 n$ dotriacontane chains) of $\dot{R}_{g y r, \gamma}$ are depicted in Fig. 14. The maximum position of the spectrum at approximately $70 \mathrm{~cm}^{-1}$ confirms that this frequency range corresponds really to the intramolecular breathing motions in the simulations, whether the atomic or the molecular scaling method is used. It is also clear from the figure that this frequency is more sharply peaked for the atomic scaling method. By comparison with Fig. 12, we conclude that the atomic scaling method couples the pressure really to these intramolecular breathing modes, while the molecular scaling method does not. As a consequence, intramolecular stress is less efficiently dissipated if the molecular scaling method is applied leading to the discrepancy between atomic and molecular pressure for the long chains described above. It seems reasonable that the low-frequency breathing modes are in turn responsible for the extremely slow relaxation of energies since conformational rearrangements are coupled to these motions. For the shorter $n$-decanes the intramolecular modes correspond to considerably higher frequencies and correspondingly motions with smaller amplitudes. Although Fig. 11 shows clearly that the atomic barostat couples also to these motions, they are probably less important for the relaxation of the system since they have only a small amplitude. It is important to let a large number of low-frequency-largeamplitude motions participate in the relaxation process.

\section{SUMMARY AND CONCLUSION}

We presented a comparison of two different constant pressure simulation techniques for molecular systems subject to geometrical constraints. The simulations showed that the atomic scaling method leads to an efficient relaxation of intramolecular stress, in particular for systems consisting of large molecules. Differences from the standard center-ofmass scaling procedure to adjust the pressure were found to be less pronounced for a liquid of relatively small $n$-decanes. However, for the longer 32 carbon chains pressure adjustment by center-of-mass scaling did not work at all. While the atomic scaling procedure is able to adjust both pressures, the atomic pressure and the molecular pressure, to the prescribed equilibrium value of 1 atm, the molecular scaling method fails to do so. An explanation for these findings was provided by an analysis of the frequency-dependent heat dissipation mechanisms. We showed that especially large concerted (low-frequency) motions determine the time scale for intramolecular relaxation processes. The simulated lowfrequent breathing modes of the $n$-dotriacontane chains were found to be in good agreement with inelastic neutron scattering experiments, where the frequency range $70-80 \mathrm{~cm}^{-1}$ was assigned to accordion-like motions of the chains. These motions were shown to be essential for the relaxation process in the system. We believe that our findings are also of importance for biomolecular simulations, where often one single protein or at most a few of them are simulated in water as solvent. It is certainly desirable that the intramolecular protein motion is on equal footing with respect to pressure control, as in reality. 


\section{APPENDIX}

\section{Constraint NPT algorithm}

In the following we sketch a numerical algorithm suitable for integrating the equations of motion (3)-(7). This concerns particularly the special features of the equations of motion discussed in Sec. II A 1. The algorithm is based on the velocity version of the Verlet algorithm, which may be formulated as

$$
\begin{gathered}
x(t+\Delta)=x(t)+\Delta \dot{x}(t)+\frac{\Delta^{2}}{2} \ddot{x}(t)+O\left(\Delta^{3}\right), \\
\dot{x}(t+\Delta)=\dot{x}(t)+\frac{\Delta}{2}[\ddot{x}(t)+\ddot{x}(t+\Delta)]+O\left(\Delta^{3}\right) .
\end{gathered}
$$

An advantage of the velocity Verlet algorithm is that both coordinates and velocities are available at the same time. In the framework of the velocity Verlet integrator constraints are mostly treated using the RATTLE scheme [27]. Here we apply a slightly different variant of the RATTLE algorithm suitable for the special requirements of the atomic scaling procedure discussed in Sec. II A 1. For the following it is helpful to write down a second-order differential equation for the Cartesian coordinates $\ddot{r}_{\gamma}^{i} \quad(\gamma=1, \ldots, K ; i$ $=1, \ldots, 3 n)$. (The index $\gamma$, which labels the molecules, will be skipped in the following to keep the formulas short. When we refer to single coordinates, e.g., $r^{i}$, the mass of the corresponding atom will be denoted as $m_{i}$.) From the equations of motion (3)-(7) we obtain

$$
\ddot{r}^{i}=\frac{d}{d t}\left(\frac{\dot{V}}{3 V}\left\{r^{i}+\frac{1}{m_{i}} \sum_{\alpha} \frac{\partial \sigma^{\alpha}}{\partial r^{i}} g_{\alpha}\right\}\right)+\frac{\dot{p}_{i}}{m_{i}},
$$

where the $g_{\alpha}$ are Lagrangian multipliers which are the solutions of the linear system of equations $\mathbf{A} \mathbf{M}^{-1} \mathbf{A}^{T} \mathbf{g}=-\mathbf{A r}$. From Eq. (A3) it is already clear that the time derivatives of the Lagrangian multipliers $g_{\alpha}$ are needed too. However, instead of constructing an equation of motion for the $g_{\alpha}$ it is easier to circumvent this problem by reformulating the accelerations $\ddot{r}^{i}$. After some algebra (see Sec. 2 of the Appendix) we find

$$
\begin{aligned}
& \ddot{r^{i}}=\left(\frac{\dot{p}_{V}}{3 W_{V} V}-\frac{2 p_{V}^{2}}{9 W_{V}^{2} V^{2}}\right) r^{i}+\frac{1}{m_{i}} \sum_{\alpha} \frac{\partial \sigma^{\alpha}}{\partial r^{i}} \beta_{\alpha} \\
&+\frac{1}{m_{i}}\left(f_{i}-\zeta p_{i}-\frac{p_{V}^{2}}{9 W_{V}^{2} V^{2}} \sum_{\alpha} g_{\alpha} \sum_{k} \frac{\partial^{2} \sigma^{\alpha}}{\partial r^{i} \partial r^{k}} r_{\perp}^{k}\right) \\
& \equiv \ddot{r}^{* i}+\frac{1}{m_{i}} \sum_{\alpha} \frac{\partial \sigma^{\alpha}}{\partial r^{i}} \beta_{\alpha} .
\end{aligned}
$$

The last equality in Eq. (A4) defines the auxiliary quantities $\ddot{r} * i$, which are useful in describing the algorithm. $r_{\perp}^{i}=r^{i}$ $-r_{\|}^{i}=-\left(1 / m_{i}\right) \Sigma_{\alpha} g_{\alpha}\left(\partial \sigma^{\alpha} / \partial r^{i}\right)$ is the part of the position vector that is orthogonal to the constraint surface. It should be noted that the $\beta_{\alpha}$ are only abbreviations that stand for

$$
\begin{aligned}
\beta_{\alpha}= & \left(\frac{\dot{p}_{V}}{3 W_{V} V}-\frac{2 p_{V}^{2}}{9 W_{V}^{2} V^{2}}\right) g_{\alpha} \\
& +\frac{p_{V}}{3 W_{V} V} \dot{g}_{\alpha}+\mu_{\alpha}-\left(\frac{p_{V}}{3 W_{V} V}\right)^{2} n_{\alpha} g_{\alpha} .
\end{aligned}
$$

$\mu_{\alpha}$ is the Lagrangian multiplier associated with the position constraints $z_{i}=\Sigma_{\alpha} \mu_{\alpha} \partial \sigma^{\alpha} / \partial r^{i}$ and $n_{\alpha}$ denotes the degree of homogeneity of the constraint gradient $\alpha$, e.g., bond constraints whose gradients are of the form $\partial \sigma^{\alpha} / \partial \mathbf{r}^{(\alpha, 1)}=\mathbf{r}^{(\alpha, 1)}$ $-\mathbf{r}^{(\alpha, 2)}$ correspond to $n_{\alpha}=1$. Instead of constructing an analytical formula for the $\dot{g}_{\alpha}$ that appear in Eq. (A5), the complete expression for $\beta_{\alpha}$ can be determined numerically in such a way that the constraints are exactly fulfilled up to a predefined tolerance. Having established the equation for $\ddot{r}^{i}$, we can sketch the algorithm for integrating the equations of motion (3)-(7) in eight steps, supposing the following quantities are available at time $t$ : input arrays

$$
\begin{gathered}
V(t), \mathbf{r}(t), \mathbf{f}(\mathbf{r}(t)), \quad \mathbf{r}_{\|}(t)=\mathbf{r}(t)-\mathbf{r}_{\perp}(t), \\
\mathbf{r}_{\perp}(t)=-\mathbf{M}^{-1} \sum_{\alpha} g_{\alpha}(t) \frac{\partial \sigma^{\alpha}}{\partial \mathbf{r}}, \\
\mathbf{p}(t), p_{V}(t), \zeta(t), \mathbf{z}(t)=\sum_{\alpha} \frac{\partial \sigma^{\alpha}}{\partial \mathbf{r}} \mu_{\alpha}(t), \\
P_{\text {inst }}^{a t m}(t)=\left\{\left(\mathbf{p}^{T} \mathbf{M}^{-1} \mathbf{p}\right)(t)+\mathbf{r}^{T}(t)[\mathbf{f}(t)+\mathbf{z}(t)]\right\} / 3 V(t) .
\end{gathered}
$$

Determine first positionlike quantities at the new time $t$ $+\Delta$.

(i) Compute the time integral of the Nosé-Hoover friction

$$
\begin{aligned}
\int_{0}^{t+\Delta} d t^{\prime} \zeta\left(t^{\prime}\right)= & \int_{0}^{t} d t^{\prime} \zeta\left(t^{\prime}\right)+\Delta \zeta(t)+\frac{\Delta^{2}}{2 W_{S}}\left(\left(\mathbf{p}^{T} \mathbf{M}^{-1} \mathbf{p}\right)(t)\right. \\
& \left.+\frac{p_{V}^{2}(t)}{W_{V}}-(f+1) k_{B} T\right) .
\end{aligned}
$$

(ii) Compute the volume

$$
V(t+\Delta)=V(t)+\Delta \frac{p_{V}(t)}{W_{V}}+\frac{\Delta^{2}}{2 W_{V}} \dot{p}_{V}(t)
$$

where $\quad \dot{p}_{V}(t)=P_{\text {inst }}^{\text {atm }}(t)-P_{\text {ext }}-\zeta(t) p_{V}(t)$.

(iii) Compute the positions

$$
\begin{aligned}
r^{i}(t+\Delta)= & r^{i}(t)+\Delta\left(\frac{p_{i}(t)}{m_{i}}+\frac{p_{V}(t)}{3 W_{V} V(t)} r_{\|}^{i}(t)\right) \\
& +\frac{\Delta^{2}}{2} \ddot{r} * i(t)+\frac{\Delta^{2}}{2 m_{i}} \sum_{\alpha} \frac{\partial \sigma^{\alpha}}{\partial r^{i}} \beta_{\alpha}(t),
\end{aligned}
$$

where $\beta_{\alpha}(t)$ can be determined similarly as in the wellknown SHAKE algorithm [13]. We recall that the $\beta_{\alpha}$ are only convenient abbreviations. They cannot be used in propagat- 
ing the momenta. The constraint forces $z_{i}(t)$ $=\Sigma_{\alpha} \mu_{\alpha}(t)\left(\partial \sigma^{\alpha} / \partial r^{i}\right)$ are determined by the $\mu_{\alpha}(t)$ and not by $\beta_{\alpha}(t)$. It is therefore necessary to find another way to obtain the $\mu_{\alpha}(t)$ for propagating the particle momenta $p_{i}$ and the momentum $p_{V}$. However, since we have not determined numerical estimates for $\mu_{\alpha}(t)$ by controlling all positions constraints in step (iii), we cannot use the standard RATTLE integrator. An important observation in the RATTLE integration scheme for constrained systems is that the local error made by determining only numerical estimates for the Lagrangian multipliers $\mu_{\alpha}(t)$ is of the same order in the time step $\Delta$ as already inherent in the velocity Verlet algorithm. In this appendix we prove that the alternative proposed here [see the comment below step (vi)] fulfills that condition too.

(iv) Use the positions to compute forces $\mathbf{f}_{i}(t+\Delta)$ and the contribution from the potential forces to the atomic virial as $\sum_{i} \mathbf{f}_{i} \cdot \mathbf{r}_{i}=\sum_{i<j} \mathbf{f}_{i j} \cdot \mathbf{r}_{i j}^{N I}$, where $\mathbf{r}_{i j}^{N I}$ denotes the threedimensional nearest image distance vector between atom $i$ and atom $j$.

(v) Compute the parallel projections $\mathbf{r}_{\|}(t+\Delta)$ or, equivalently, the multipliers $g_{\alpha}$ in $\mathbf{r}_{\perp}(t+\Delta)=\left(\mathbf{r}-\mathbf{r}_{\|}\right)(t+\Delta)=$ $-\sum_{\alpha} g_{\alpha}(t+\Delta) \mathbf{M}^{-1}\left(\partial \sigma^{\alpha} / \partial \mathbf{r}\right)(t+\Delta)$. The determination of the $g_{\alpha}(t+\Delta)$ can be performed numerically by the same routine that is used in the RATTLE algorithm [27] to fulfill velocity constraints of the form $\mathbf{A r}=\mathbf{O}$. Here the constraints read instead $\mathbf{A} \mathbf{r}_{\|}=\mathbf{A r}-\mathbf{A} \mathbf{r}_{\perp}=\mathbf{O}$; compare Eq. (11).

(vi) Determine the momenta $\mathbf{p}(t+\Delta), p_{V}(t+\Delta)$, and $\zeta(t+\Delta)$ iteratively: Compute first $\mathbf{p}(t+\Delta / 2), p_{V}(t+\Delta / 2)$, and $\zeta(t+\Delta / 2)$, which are only propagated half a time step $\Delta / 2$ and can be used to determine a zeroth approximation for the full time step propagated quantities

$$
\begin{aligned}
p_{i}^{(0)}(t+\Delta)= & p_{i}(t)+\Delta\left[p_{i}(t+\Delta / 2)-p_{i}(t)\right], \\
p_{i}(t+\Delta / 2)= & p_{i}(t)+\frac{\Delta}{2}\left\{f_{i}(t)+z_{i}(t)-\zeta(t) p_{i}(t)-\frac{p_{V}(t)}{3 W_{V} V(t)}\right. \\
& \left.\times\left(p_{i}(t)+\sum_{\alpha, k} g_{\alpha}(t) \frac{\partial^{2} \sigma^{\alpha}}{\partial r^{i} \partial r^{k}} \frac{p_{k}(t)}{m_{k}}\right)\right\} .
\end{aligned}
$$

It should be noted that in the case of bond constraints only the second derivatives $\partial^{2} \sigma^{\alpha} / \partial r^{i} \partial r^{k}$ are simply constants:

$$
\begin{gathered}
p_{V}^{(0)}(t+\Delta)=p_{V}(t)+\Delta\left[p_{V}(t+\Delta / 2)-p_{V}(t)\right] \\
p_{V}(t+\Delta / 2)=p_{V}(t)+\frac{\Delta}{2}\left[P_{\text {inst }}^{\text {atm }}(t)-P_{\text {ext }}-\zeta(t) p_{V}(t)\right] \\
\zeta^{(0)}(t+\Delta)=\zeta(t)+\Delta[\zeta(t+\Delta / 2)-\zeta(t)] \\
\zeta(t+\Delta / 2)=\zeta(t)+\frac{\Delta}{2 W_{S}}\left(\left(\mathbf{p}^{T} \mathbf{M}^{-1} \mathbf{p}\right)(t)\right. \\
\left.+\frac{p_{V}^{2}}{W_{V}}(t)-(f+1) k_{B} T\right)
\end{gathered}
$$

As already mentioned, the constraint forces $z_{i}(t)$ and the constraint virial at time $t$ were not determined in step (iii). They had to be determined in the previous time step cycle $t-\Delta \rightarrow t$ as described in (vii). If there is no previous cycle, i.e., the program runs the first time step, one may start with the approximation $\mu_{\alpha}(0)=\beta_{\alpha}(0)$.

(vii) Start iterations $\nu=0, \ldots, \nu_{\text {max }}$ :

$$
\begin{aligned}
\zeta^{(\nu+1)}(t+\Delta)= & \zeta(t+\Delta / 2)+\frac{\Delta}{2 W_{S}}\left(\left(\mathbf{p}^{(\nu) T} \mathbf{M}^{-1} \mathbf{p}^{(\nu)}\right)(t+\Delta)\right. \\
& \left.+\frac{p_{V}^{(\nu) 2}(t+\Delta)}{W_{V}}-(f+1) k_{B} T\right) \\
p_{i}^{(\nu+1)}(t+\Delta)= & p_{i}(t+\Delta / 2) \\
& +\frac{\Delta}{2}\left\{f_{i}(t+\Delta)-\zeta^{(\nu+1)}(t+\Delta) p_{i}^{(\nu)}(t+\Delta)\right. \\
& -\frac{p_{V}^{(\nu)}(t+\Delta)}{3 W_{V} V(t+\Delta)}\left(p_{i}^{(\nu)}(t+\Delta)\right. \\
& \left.\left.+\sum_{\alpha, k} g_{\alpha}(t+\Delta) \frac{\partial^{2} \sigma^{\alpha}}{\partial r^{i} \partial r^{k}} \frac{p_{k}^{(\nu)}(t+\Delta)}{m_{k}}\right)\right\} \\
& +\frac{\Delta}{2} \sum_{\alpha} \frac{\partial \sigma^{\alpha}}{\partial r^{i}}(t+\Delta) \mu_{\alpha}^{(\nu)}(t+\Delta),
\end{aligned}
$$

where $\mu_{\alpha}^{(\nu)}(t+\Delta)$ are the Lagrangian multipliers corresponding to the $\nu$ th approximation to the constraint forces $\mathbf{z}^{(\nu)}(t+\Delta)$. In each iteration these estimates can be determined using a routine as described in the RATTLE algorithm. Determine an estimate for the atomic pressure at time $t+\Delta$ :

$$
\begin{aligned}
P_{\text {inst }}^{(\nu) a t m}(t+\Delta)= & \frac{1}{3 V(t+\Delta)}\left\{\left(\mathbf{p}^{(\nu+1) T} \mathbf{M}^{-1} \mathbf{p}^{(\nu+1)}\right)(t+\Delta)\right. \\
& \left.+\sum_{i} r^{i}(t+\Delta)\left[f_{i}(t+\Delta)+z_{i}^{(\nu)}(t+\Delta)\right]\right\}, \\
p_{V}^{(\nu+1)}(t+\Delta)= & p_{V}(t+\Delta / 2)+\frac{\Delta}{2}\left[-P_{\text {ext }}+P_{\text {inst }}^{(\nu) a t m}(t+\Delta)\right. \\
& \left.-\zeta^{(\nu+1)}(t+\Delta) p_{V}^{(\nu)}(t+\Delta)\right] .
\end{aligned}
$$

The respective estimate for the constraint virial $\Sigma_{i} \mathbf{r}_{i} \cdot \mathbf{z}_{i}^{(\nu)}$ can be computed from the estimates for the Lagrange multipliers $\mu_{\alpha}^{(\nu)}$ as $\Sigma_{\alpha} \mu_{\alpha}^{(\nu)} d^{\alpha 2}$, where $d^{\alpha}$ is the fixed bond length in $\sigma^{\alpha}=\left[\left(\mathbf{r}^{(\alpha, 1)}-\mathbf{r}^{(\alpha, 2)}\right)^{2}-d^{\alpha 2}\right] / 2=0$.

(viii) Store the quantities at time $t+\Delta$ in the input arrays. Store the actual time. Set $t \rightarrow t+\Delta$. Go to (i).

\section{Validity of Eq. (A4)}

In this section we prove the validity of Eq. (A4) for the particle accelerations in the case of atomic scaling. Starting with Eq. (A3) we find

$$
\begin{aligned}
\ddot{r}^{i}= & \frac{1}{3 W_{V}}\left(\frac{\dot{p}_{V}}{V}-\frac{p_{V}^{2}}{W_{V} V^{2}}\right)\left(r^{i}+\frac{1}{m_{i}} \sum_{\alpha} \frac{\partial \sigma^{\alpha}}{\partial r^{i}} g_{\alpha}\right) \\
& +\frac{p_{V}}{3 W_{V} V}\left(\dot{r}^{i}+\sum_{\alpha} \frac{g_{\alpha}}{m_{i}} \sum_{k} \frac{\partial^{2} \sigma^{\alpha}}{\partial r^{i} \partial r^{k}} \dot{r}^{k}+\sum_{\alpha} \frac{\partial \sigma^{\alpha}}{\partial r^{i}} \frac{\dot{g}_{\alpha}}{m_{i}}\right)+\frac{\dot{p}_{i}}{m_{i}} .
\end{aligned}
$$


Using the equation of motion for $\dot{r}^{k}$ [Eq. (3)], the term containing the second derivatives of the constraints can be reformulated as

$$
\begin{aligned}
& \sum_{\alpha} \frac{g_{\alpha}}{m_{i}} \sum_{k} \frac{\partial^{2} \sigma^{\alpha}}{\partial r^{i} \partial r^{k}} \dot{r}^{k} \\
& =\sum_{\alpha, k} \frac{g_{\alpha}}{m_{i}} \frac{\partial^{2} \sigma^{\alpha}}{\partial r^{i} \partial r^{k}} \frac{p_{k}}{m_{k}}+\frac{p_{V}}{3 W_{V} V}(\underbrace{\frac{g_{\alpha}}{m_{i}} n_{\alpha} \frac{\partial \sigma^{\alpha}}{\partial r^{i}}}_{\sum_{\alpha, k} \frac{g_{\alpha}}{m_{i}} \frac{\partial^{2} \sigma^{\alpha}}{\partial r^{i} \partial r^{k}} r^{k}}-\sum_{\alpha, k} \frac{g_{\alpha}}{m_{i}} \frac{\partial^{2} \sigma^{\alpha}}{\partial r^{i} \partial r^{k}} r_{\perp}^{k}),
\end{aligned}
$$

where $n_{\alpha}$ is the degree of homogeneity of the constraint gradient number $\alpha$. In the final expression for $\ddot{r}_{i}$ the first term in Eq. (A8) drops out since it appears with opposite sign in the expression for $\dot{p}_{i} / m_{i}$ in Eq. (A7). Using Eq. (A8), the equations of motion (3) and (4) for $r_{i}$ and $p_{i}$ in Eq. (A7), and reordering of the terms yields Eq. (A4). All coefficients in front of $\partial \sigma^{\alpha} / \partial r^{i}$ are collected in the abbreviations $\beta_{\alpha}$ as described in Eq. (A5).

\section{Local error}

Following Andersen's arguments in [27] quite closely, we prove here that the local error of the coordinates and of the velocities for the proposed constraint algorithm is of the same order $\Delta^{3}$ as is the error principally inherent in the velocity version of the Verlet algorithm. Concentrating on the Cartesian coordinates $r^{i}$, their parallel projections $r_{\|}^{i}$, and the momenta $p_{i}$ of the molecular system, our algorithm can be summarized as

$$
\begin{gathered}
r^{i}(t+\Delta)=r^{i}(t)+\Delta\left(\frac{p_{i}(t)}{m_{i}}+\frac{p_{V}(t)}{3 W_{V} V(t)} r_{\|}^{i}(t)\right) \\
+\frac{\Delta^{2}}{2} \ddot{r}^{* i}(t)+\frac{\Delta^{2}}{2 m_{i}} \sum_{\alpha} \frac{\partial \sigma^{\alpha}}{\partial r^{i}}(t) \beta_{\alpha}(t), \\
r_{\|}^{i}(t+\Delta)=r^{i}(t+\Delta)+\sum_{\alpha} \frac{g_{\alpha}(t+\Delta)}{m_{i}} \frac{\partial \sigma^{\alpha}}{\partial r^{i}}(t+\Delta), \\
p_{i}(t+\Delta)=p_{i}(t)+\frac{\Delta}{2}\left(\dot{p}_{i}^{*}(t)+\sum_{\alpha} \mu_{\alpha}(t) \frac{\partial \sigma^{\alpha}}{\partial r^{i}}(t)\right. \\
\left.+\dot{p}_{i}^{*}(t+\Delta)+\sum_{\alpha} \mu_{\alpha}(t+\Delta) \frac{\partial \sigma^{\alpha}}{\partial r^{i}}(t+\Delta)\right)
\end{gathered}
$$

where $\ddot{r}^{* i}$ is the abbreviation introduced in Eq. (A4) and $\dot{p}_{i}^{*}$ stands for

$$
\dot{p}_{i}^{*}=f_{i}-\zeta p_{i}-\frac{p_{V}}{3 W_{V} V}\left(p_{i}+\sum_{\alpha, k} g_{\alpha} \frac{\partial^{2} \sigma^{\alpha}}{\partial r^{i} \partial r^{k}} \frac{p_{k}}{m_{k}}\right)
$$

In Eq. (A9) the help quantity $\ddot{r}^{* i}$ is completely known at time $t$. When instead of the numerical estimates $\beta_{\alpha}$ the exact values at time $t$ were used in Eq. (A9) the geometrical constraints would be possibly violated due to local errors in the velocity Verlet integrator. At worst, this violation would be of $O\left(\Delta^{3}\right)$. Since the numerical estimates $\beta_{\alpha}$ are approximations for the exact values that are multiplied by $\Delta^{2}$ in Eq. (A9) and since they guarantee that the constraints are exactly fulfilled, they deviate from the exact values by $O(\Delta)$. Therefore, the possible errors in the positions $r^{i}(t+\Delta)$ are still of $O\left(\Delta^{3}\right)$.

Since the parallel projection in Eq. (A10) is a purely geometrical operation that is independent of the time step, the multipliers $g_{\alpha}(t+\Delta)$ involve the same local error as the positions themselves, namely, $O\left(\Delta^{3}\right)$. Thus, if the momenta are correct up to $O\left(\Delta^{3}\right)$ (this will be shown next), the velocities will also involve an error of the same order. If the time step is small enough, it can be ensured that the iterative procedure in step (vii) of the algorithm will converge, such that the final iteration will give a propagation of the momenta that is equivalent to the formula (A11). Thus $p_{i}^{*}(t)$ and $p_{i}^{*}(t+\Delta)$ in Eq. (A11) can be assumed to be correct up to a local error of $O\left(\Delta^{3}\right)$. If $\mu_{\alpha}(t+\Delta)$ were replaced by the exact (but unknown) Lagrangian multipliers the momentum constraints would be possibly violated and the local error would be of $O\left(\Delta^{3}\right)$. We can therefore conclude that the $\mu_{\alpha}(t+\Delta)$ need to deviate from the exact values by $O\left(\Delta^{2}\right)$ in order to fulfill the momentum constraints exactly since they are multiplied by a factor proportional to $\Delta$. The same holds consequently for $\mu_{\alpha}(t)$ determined in the previous time step. These arguments prove that no larger local error than already inherent in the velocity Verlet algorithm will be introduced when the proposed integration scheme (i) - (viii) is used. It is worth noting that the estimates for the Lagrangian multipliers $\mu_{\alpha}$ are correct up to an error $O\left(\Delta^{2}\right)$. This is the minimal needed accuracy, since the Lagrangian multipliers enter directly the atomic pressure. If the Lagrangian multipliers were obtained with lower accuracy the local error in the propagation of $p_{V}(t+\Delta)=p_{V}(t+\Delta / 2)+\Delta / 2\left\{P_{i n s t}^{a t m}(t+\Delta)-P_{\text {ext }}\right.$ $\left.-\zeta(t+\Delta) p_{V}(t+\Delta)\right\}$ would be larger than the inherent local error of the velocity Verlet integrator. 
[1] H. C. Andersen, J. Chem. Phys. 72, 2384 (1980).

[2] S. Nosé, J. Chem. Phys. 81, 511 (1984).

[3] G. R. Kneller and T. Mülders, Phys. Rev. E 54, 6825 (1996).

[4] S. Melchionna and G. Ciccotti, J. Chem. Phys. 106, 195 (1997).

[5] G. R. Kneller and T. Mülders (unpublished).

[6] J.-P. Ryckaert and G. Ciccotti, Mol. Phys. 58, 1125 (1986).

[7] G. Ciccotti and J.-P. Ryckaert, Comput. Phys. Rep. 4, 345 (1986).

[8] J.-P. Ryckaert and G. Ciccotti, J. Chem. Phys. 78, 7368 (1983).

[9] M. Ferrario, in Computer Simulation in Chemical Physics, Vol. 153 of NATO Advanced Study Institute, Series B: Physics, edited by M. P. Allen and D. J. Tildesley (Kluwer, Dodrecht, 1993).

[10] M. Ferrario and J.-P. Ryckaert, Mol. Phys. 54, 587 (1985).

[11] W. F. van Gunsteren and H. J. C. Berendsen, Angew. Chem. Int. Ed. Engl. 29, 929 (1990).

[12] P. A. M. Dirac, Can. J. Math. 2, 129 (1950); Proc. R. Soc. London, Ser. A 246, 326 (1958); Lectures on Quantum Mechanics (Yeshiva University, New York, 1974).

[13] J.-P. Ryckaert, G. Ciccotti, and H. J. C. Berendsen, J. Comput. Phys. 23, 327 (1977).

[14] R. Kubo, M. Toda, and N. Hashitsume, in Statistical Physics II, 2nd ed., edited by P. Fulde, Springer Series in Solid-State
Sciences Vol. 31 (Springer, Berlin, 1991).

[15] R. Becker, Theorie der Wärme, 3rd ed. (Springer, Berlin, 1985).

[16] W. Smith, Inf. Q. Comput. Simulat. Condens. Phases 39, 14 (1993).

[17] D. J. Evans and B. L. Holian, J. Chem. Phys. 83, 4069 (1985).

[18] D. J. Evans, in Molecular-Dynamics Simulation of StatisticalMechanical System, Proceedings of the International School of Physics XCVII, edited by G. Ciccotti and W. G. Hoover (Elsevier, Amsterdam, 1986), p. 221.

[19] S. Toxvaerd, J. Chem. Phys. 93, 4290 (1990).

[20] G. D. Smith and R. L. Jaffe, J. Phys. Chem. 100, 18718 (1996).

[21] S. Toxvaerd, J. Chem. Phys. 107, 5197 (1997).

[22] M. P. Allen and D. J. Tildesley, Computer Simulation of Liquids (Oxford University Press, Oxford, 1987).

[23] W. F. van Gunsteren and A. E. Mark, J. Chem. Phys. 108, 6109 (1998).

[24] A. Arni, Grundkurs Chemie II (VCH, Weinheim, 1995).

[25] S. Nosé and M. Klein, Mol. Phys. 50, 1055 (1983).

[26] CRC Handbook of Chemistry and Physics, 58th ed., edited by R. C. Weast (CRC, Cleveland, 1977).

[27] H. C. Andersen, J. Comput. Phys. 52, 24 (1983).

[28] K. Kaji, A. Kollmar, B. Ewen, R. Stockmeyer, and E. W. Fischer, J. Appl. Phys. 52, 6137 (1981). 